\title{
Borane-Catalyzed Transfer Hydrogenations of Pyridines with Ammonia Borane
}

Qiwen Zhou, ${ }^{\dagger}$ Lanqiong Zhang, ${ }^{\dagger}$ Wei Meng, ${ }^{\ddagger, \S}$ Xiangqing Feng ${ }^{\ddagger}{ }^{\ddagger}$ Jing Yang, ${ }^{*, \dagger}$ and

$$
\text { Haifeng } \mathrm{Du}^{*}, \S
$$

${ }^{\dagger}$ State Key Laboratory of Chemical Resource, Beijing Key Laboratory of Bioprocess, College of Life Science and Technology, Beijing University of Chemical Technology, Beijing 100029, China

* Beijing National Laboratory for Molecular Sciences, CAS Key Laboratory of Molecular Recognition and Function, Institute of Chemistry, Chinese Academy of Sciences, Beijing 100190, China

${ }^{\S}$ University of Chinese Academy of Sciences, Beijing 100049, China

\section{Supporting Information}

\section{Table of contents}

$\begin{array}{ll}\text { Representative procedure for hydrogenation of pyridines } & \text { S2 }\end{array}$

$\begin{array}{ll}{ }^{11} \text { B NMR study on the reaction mixture } & \text { S2 }\end{array}$

$\begin{array}{ll}\text { Characterization of product } & \text { S3 }\end{array}$

$\begin{array}{ll}\text { Copies of NMR spectra } & \text { S11 }\end{array}$ 
General information: All air-sensitive compounds were handled under an atmosphere of argon or in a nitrogen-filled glovebox. ${ }^{1} \mathrm{H}$ NMR and ${ }^{13} \mathrm{C}$ NMR spectra were recorded on Bruker AV 400 at ambient temperature with $\mathrm{CDCl}_{3}$ as solvent and TMS as internal standard. Chemical shifts $(\delta)$ were given in ppm, referenced to the residual proton resonance of TMS (0), to the carbon resonance of the $\mathrm{CDCl}_{3}(77.23)$. Coupling constants $(J)$ were given in Hertz $(\mathrm{Hz})$. IR spectrums were recorded on Perkin-Elmer-983 spectrometer. Column chromatography was performed on silica gel (200-300 mesh). All solvents were purified by conventional methods, distilled before use.

Representative procedure for hydrogenation of pyridines (Scheme 3, 5a): To a sealed tube (15 mL), were added 2,6-diphenylpyridine (3a) (0.0924 g, $0.40 \mathrm{mmol})$, $\mathrm{B}\left(\mathrm{C}_{6} \mathrm{~F}_{5}\right)_{3}(0.0205 \mathrm{~g}, 0.040 \mathrm{mmol})$, ammonia borane (0.0497 $\left.\mathrm{g}, 1.6 \mathrm{mmol}\right)$, and dry toluene $(1.0 \mathrm{~mL})$ in a nitrogen atmosphere glovebox. The resulting mixture was stirred at $120{ }^{\circ} \mathrm{C}$ for $6 \mathrm{~h}$. After cooling to ambient temperature, the solvent was removed under reduced pressure. The crude residue was purified by column chromatography on silica gel using petroleum ether/ethyl acetate $(10 / 1)$ to petroleum ether/ethyl acetate/ $\mathrm{Et}_{3} \mathrm{~N}(5 / 1 / 0.1)$ as the eluent to give 2,6-diphenylpiperidine (5a) as a white solid (0.0768 g, 81\% yield).

${ }^{11}$ B NMR study on the reaction mixture: After the solvent of the reaction mixture was removed under reduced pressure, the crude residue was dissolved in $\mathrm{C}_{6} \mathrm{D}_{6}(2.0$ mL). $\mathrm{A}^{11} \mathrm{~B}$ NMR spectra is recorded on Bruker AV 400 at room temperature, and the 
chemical shift is relative to $\mathrm{Et}_{2} \mathrm{O} \cdot \mathrm{BF}_{3}(0 \mathrm{ppm})$.
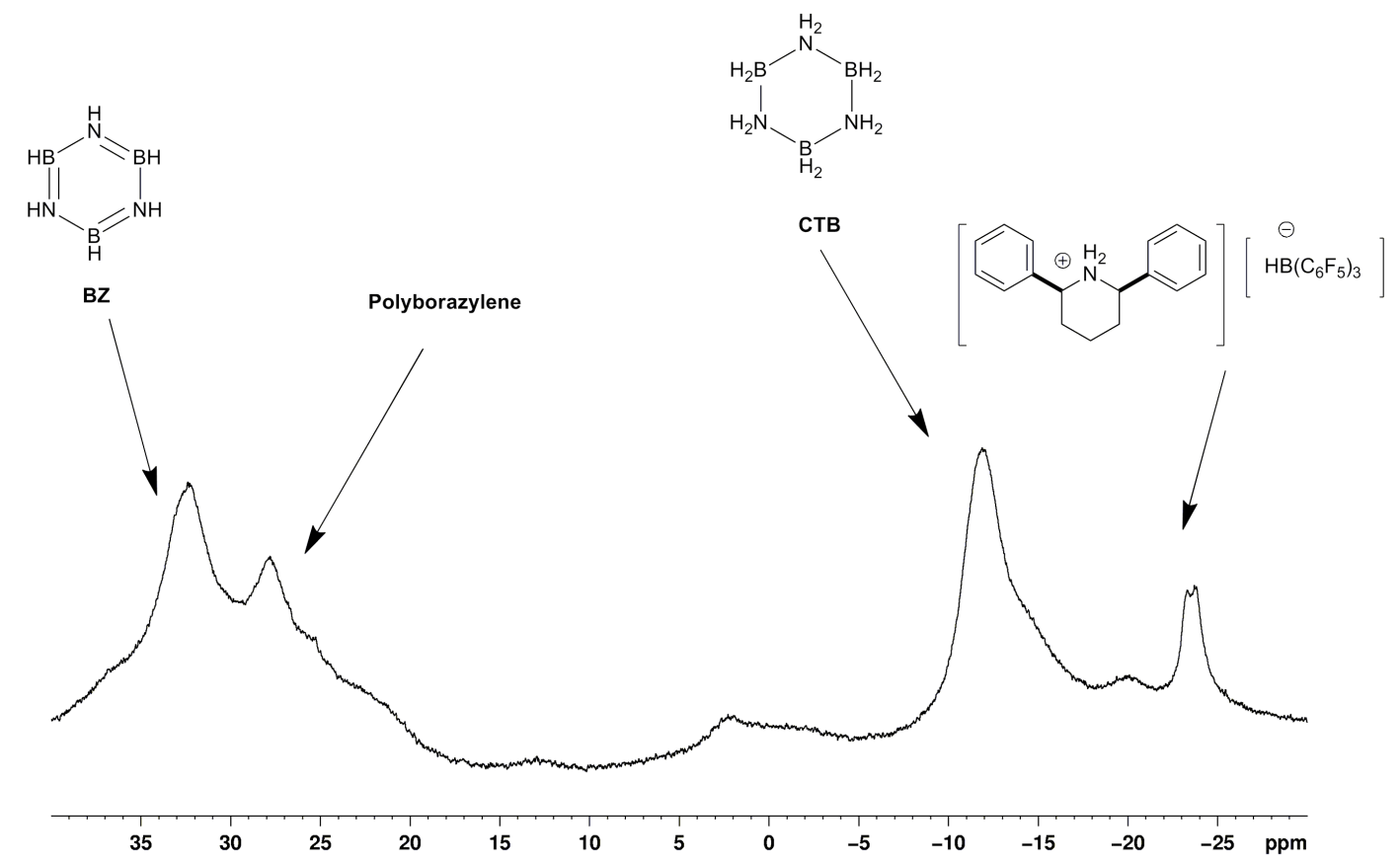

Figure S1<smiles>c1ccc(C2CCCC(c3ccccc3)N2)cc1</smiles>

Cis-2,6-diphenylpiperidine (5a): 0.0765 g, 81\% yield, cis/trans = 99/1, white solid; ${ }^{1} \mathrm{H}$ NMR (400 MHz, $\left.\mathrm{CDCl}_{3}, \mathrm{ppm}\right): \delta 7.49(\mathrm{~d}, J=7.6 \mathrm{~Hz}, 4 \mathrm{H}), 7.40-7.32(\mathrm{~m}, 4 \mathrm{H})$, 7.30-7.24 (m, 2H), 3.88-3.82 (m, 2H), 2.06-1.96 (m, 1H), 1.90-1.82 (m, 3H), 1.76-1.49 (m, 3H); $\left.{ }^{13} \mathrm{C} \mathrm{NMR} \mathrm{(100} \mathrm{MHz,} \mathrm{CDCl}_{3}, \mathrm{ppm}\right): \delta$ 146.0, 128.5, 127.2, 126.9, $62.8,34.9,26.0$.

Liu, Y.; Du, H. J. Am. Chem. Soc. 2013, 135, 12968.<smiles>Cc1ccc(C2CCCC(c3ccc(C)cc3)N2)cc1</smiles> 
Cis-2,6-di(p-tolyl)piperidine (5b): 0.0806 g, 76\% yield, cis/trans = 97/3, white solid; ${ }^{1} \mathrm{H}$ NMR (400 MHz, $\left.\mathrm{CDCl}_{3}, \mathrm{ppm}\right): \delta 7.33(\mathrm{~d}, J=8.0 \mathrm{~Hz}, 4 \mathrm{H}), 7.11(\mathrm{~d}, J=8.0 \mathrm{~Hz}$, 4H), 3.80-3.69 (m, 2H), $2.32(\mathrm{~s}, 6 \mathrm{H}), 2.00-1.88(\mathrm{~m}, 1 \mathrm{H}), 1.86-1.73(\mathrm{~m}, 3 \mathrm{H}), 1.70-1.44$ (m, 3H); ${ }^{13} \mathrm{C}$ NMR $\left(100 \mathrm{MHz}, \mathrm{CDCl}_{3}, \mathrm{ppm}\right): \delta 143.2,136.7,129.2,126.8,62.6,35.0$, 26.1, 21.3 .

Liu, Y.; Du, H. J. Am. Chem. Soc. 2013, 135, 12968.<smiles>COc1ccc(C2CCCC(c3ccc(OC)cc3)N2)cc1</smiles>

Cis-2,6-bis(4-methoxyphenyl)piperidine (5c): $0.0749 \mathrm{~g}, 63 \%$ yield, cis/trans $=99 / 1$, white solid; ${ }^{1} \mathrm{H}$ NMR (400 MHz, $\left.\mathrm{CDCl}_{3}, \mathrm{ppm}\right): \delta 7.35$ (d, $\left.J=8.4 \mathrm{~Hz}, 4 \mathrm{H}\right), 6.84(\mathrm{~d}, J=$ $8.4 \mathrm{~Hz}, 4 \mathrm{H}), 3.70-3.70(\mathrm{~m}, 8 \mathrm{H}), 2.00-1.88(\mathrm{~m}, 1 \mathrm{H}), 1.88-1.69(\mathrm{~m}, 3 \mathrm{H}), 1.66-1.40(\mathrm{~m}$, $3 \mathrm{H}) ;{ }^{13} \mathrm{C}$ NMR $\left(100 \mathrm{MHz}, \mathrm{CDCl}_{3}, \mathrm{ppm}\right): \delta 158.7,138.3,127.9,113.8,62.2,55.4$, $34.9,26.0$.

Liu, Y.; Du, H. J. Am. Chem. Soc. 2013, 135, 12968.<smiles>Fc1ccc(C2CCCC(c3ccc(F)cc3)N2)cc1</smiles>

Cis-2,6-bis(4-fluorophenyl)piperidine (5d): 0.0930 g, 85\% yield, cis/trans $=99 / 1$, white solid, m.p. $179-181{ }^{\circ} \mathrm{C}$; IR (film): 3268, 1514, $1465 \mathrm{~cm}^{-1} ;{ }^{1} \mathrm{H}$ NMR (400 MHz, $\left.\mathrm{CDCl}_{3}, \mathrm{ppm}\right): \delta$ 7.29-7.16 (m, 4H), 6.98-6.83 (m, 4H), 4.03-3.91 (m, 2H), 2.12-2.00 (m, 3H), 1.99-1.70 (m, 4H); ${ }^{13} \mathrm{C}$ NMR (100 MHz, $\left.\mathrm{CDCl}_{3}, \mathrm{ppm}\right): \delta 162.6\left(\mathrm{~d}, J_{C-F}=\right.$ $247.0 \mathrm{~Hz}), 135.8\left(\mathrm{~d}, J_{C-F}=3.0 \mathrm{~Hz}\right), 129.0\left(\mathrm{~d}, J_{C-F}=9.0 \mathrm{~Hz}\right), 115.6\left(\mathrm{~d}, J_{C-F}=22.0 \mathrm{~Hz}\right)$, 
68.9, 35.1, 24.0; HRMS (ESI) calcd. for $\mathrm{C}_{17} \mathrm{H}_{18} \mathrm{NF}_{2}[\mathrm{M}+\mathrm{H}]^{+}:$274.1402, Found: 274.1402 .<smiles>COc1cccc(C2CCCC(c3cccc(OC)c3)N2)c1</smiles>

Cis-2,6-bis(3-methoxyphenyl)piperidine (5e): $0.0956 \mathrm{~g}, 80 \%$ yield, cis/trans $=99 / 1$, colorless oil; ${ }^{1} \mathrm{H}$ NMR (400 MHz, $\left.\mathrm{CDCl}_{3}, \mathrm{ppm}\right): \delta$ 7.29-7.19 (m, 2H), 7.09-6.98 (m, 4H), 6.83-6.75 (m, 2H), 3.91-3.82 (m, 8H), 1.99-1.94 (m, 1H), 1.86-1.74 (m, 3H), 1.67-1.46 (m, 3H); ${ }^{13} \mathrm{C}$ NMR (100 MHz, $\left.\mathrm{CDCl}_{3}, \mathrm{ppm}\right): \delta 159.8,147.8,129.5,119.4$, $112.6,112.5,62.7,55.4,35.0,26.0$.

Liu, Y.; Du, H. J. Am. Chem. Soc. 2013, 135, 12968.<smiles>COc1ccc(C2CCCC(c3ccc(F)cc3)N2)cc1</smiles>

Cis-2-(4-fluorophenyl)-6-(4-methoxyphenyl)piperidine (5f): 0.0500 g, 88\% yield, cis/trans $=97 / 3$, colorless oil; ${ }^{1} \mathrm{H}$ NMR (400 MHz, $\left.\mathrm{CDCl}_{3}, \mathrm{ppm}\right): \delta$ 7.50-7.27 (m, 4H), 7.04-6.95 (m, 2H), $6.88(\mathrm{~d}, J=8.4 \mathrm{~Hz}, 2 \mathrm{H}), 3.81-3.69(\mathrm{~m}, 5 \mathrm{H}), 2.02-1.93(\mathrm{~m}, 1 \mathrm{H})$, 1.89-1.73 (m, 3H), 1.71-1.42 (m, 3H); ${ }^{13} \mathrm{C}$ NMR (100 MHz, $\left.\mathrm{CDCl}_{3}, \mathrm{ppm}\right): \delta 162.2(\mathrm{~d}$, $\left.J_{C-F}=243.0 \mathrm{~Hz}\right), 158.8,141.8\left(\mathrm{~d}, J_{C-F}=2.0 \mathrm{~Hz}\right), 138.1,128.3\left(\mathrm{~d}, J_{C-F}=8.0 \mathrm{~Hz}\right)$, $128.0,115.2\left(\mathrm{~d}, J_{C-F}=21.0 \mathrm{~Hz}\right), 113.9,62.2,62.1,55.4,35.1,34.8,25.9$.

Liu, Y.; Du, H. J. Am. Chem. Soc. 2013, 135, 12968.<smiles>c1coc(C2CCCC(c3ccco3)N2)c1</smiles> 
Cis-2,6-di(furan-2-yl)piperidine (5g): 0.0635 g, 73\% yield, cis/trans = 97/3, colorless oil; ${ }^{1} \mathrm{H}$ NMR $\left(400 \mathrm{MHz}, \mathrm{CDCl}_{3}, \mathrm{ppm}\right): \delta$ 7.37-7.28 (m, 2H), 6.32-6.25 (m, 2H), 6.20-6.14 (m, 2H), 3.94-3.85 (m, 2H), 2.21 (brs, 1H), 2.05-1.90 (m, 3H), 1.74-1.54 (m, 3H); ${ }^{13} \mathrm{C}$ NMR (100 MHz, $\left.\mathrm{CDCl}_{3}, \mathrm{ppm}\right): \delta 157.2,141.4,110.1,104.6$, $55.0,30.4,24.5$.

Liu, Y.; Du, H. J. Am. Chem. Soc. 2013, 135, 12968.

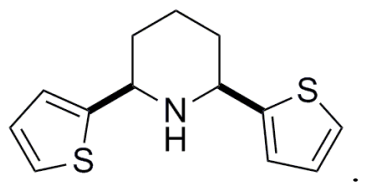

2,6-Di(thien-2-yl)piperidine (5h): 0.0757 g, 76\% yield, cis/trans = 95/5, white solid, m.p. 55-62 ${ }^{\circ} \mathrm{C}$; IR (film): 3304, 1593, 1438, $1289 \mathrm{~cm}^{-1} ;{ }^{1} \mathrm{H}$ NMR (400 MHz, $\mathrm{CDCl}_{3}$, ppm): $\delta$ 7.24-7.15 (m, 2H), $6.98(\mathrm{~d}, J=3.2 \mathrm{~Hz}, 2 \mathrm{H}), 6.96-6.92(\mathrm{~m}, 2 \mathrm{H}), 4.17-4.10(\mathrm{~m}$, 2H), 2.08 (brs, 1H), 2.02-1.95 (m, 3H), 1.69-1.55 (m, 3H); ${ }^{13} \mathrm{C}$ NMR (100 MHz, $\left.\mathrm{CDCl}_{3}, \mathrm{ppm}\right): \delta 149.4,126.5,123.8,123.0,57.9,35.4,25.4$; HRMS (ESI) calcd. for $\mathrm{C}_{13} \mathrm{H}_{16} \mathrm{NS}_{2}[\mathrm{M}+\mathrm{H}]^{+}:$250.0724, Found: 250.0719.<smiles>CC1CCCC(c2ccccc2)N1</smiles>

Cis-2-methyl-6-phenylpiperidine (5i): 0.0567 g, 81\% yield, cis/trans $=86 / 14$, colorless oil; ${ }^{1} \mathrm{H} \mathrm{NMR}\left(400 \mathrm{MHz}, \mathrm{CDCl}_{3}, \mathrm{ppm}\right): \delta 7.28(\mathrm{~d}, J=7.2 \mathrm{~Hz}, 2 \mathrm{H}), 7.26-7.18$ (m, 2H), 7.18-7.10 (m, 1H), 3.64-3.49 (m, 1H), 2.80-2.65 (m, 1H), 1.84-1.72 (m, 1H), 1.70-1.52 (m, 3H), 1.48-1.30 (m, 2H), 1.13-1.04 (m, 1H), $1.02(\mathrm{~d}, J=6.4 \mathrm{~Hz}, 3 \mathrm{H})$; ${ }^{13} \mathrm{C} \mathrm{NMR}\left(100 \mathrm{MHz}, \mathrm{CDCl}_{3}, \mathrm{ppm}\right): \delta 145.7,128.5,127.1,126.9,62.6,53.3,34.4$, 
$34.0,25.5,23.2$.

Liu, Y.; Du, H. J. Am. Chem. Soc. 2013, 135, 12968.

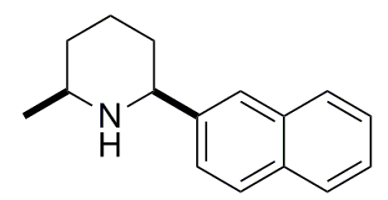

Cis-2-methyl-6-(naphthalen-2-yl)piperidine (5j): 0.0721 g, 80\% yield, cis/trans = 99/1, white solid; ${ }^{1} \mathrm{H}$ NMR (400 MHz, $\left.\mathrm{CDCl}_{3}, \mathrm{ppm}\right): \delta$ 7.85-7.73 (m, 4H), 7.52-7.46 (m, 1H), 7.46-7.37 (m, 2H), 3.86-3.73 (m, 1H), 2.90-2.74 (m, 1H), 1.97-1.75 (m, 2H), 1.74-1.61 (m, 2H), 1.60-1.43 (m, 2H), 1.28-1.16 (m, 1H), $1.13(\mathrm{~d}, J=6.4 \mathrm{~Hz}, 3 \mathrm{H})$; ${ }^{13} \mathrm{C} \mathrm{NMR}\left(100 \mathrm{MHz}, \mathrm{CDCl}_{3}, \mathrm{ppm}\right): \delta 143.2,133.7,132.9,128.0,127.9,127.7,126.0$ $125.7,125.6,124.9,62.7,53.4,34.6,34.0,25.6,23.4$.

Liu, Y.; Du, H. J. Am. Chem. Soc. 2013, 135, 12968.<smiles>CC1CCCC(c2ccc(-c3ccccc3)cc2)N1</smiles>

Cis-2-[(1,1'-biphenyl)-4-yl]-6-methylpiperidine (5k): 0.0885 g, 88\% yield, cis/trans = 99/1, colorless oil; ${ }^{1} \mathrm{H}$ NMR (400 MHz, $\left.\mathrm{CDCl}_{3}, \mathrm{ppm}\right): \delta 7.62-7.50(\mathrm{~m}, 4 \mathrm{H})$, 7.48-7.37 (m, 4H), 7.36-7.27 (m, 1H), 3.76-3.63 (m, 1H), 2.89-2.75 (m, 1H), 1.95-1.86 (m, 1H), 1.83-1.74 (m, 1H), 1.70-1.63 (m, 1H), 1.62-1.42 (m, 3H), 1.26-1.14 (m, 1H), $1.13(\mathrm{~d}, J=6.4 \mathrm{~Hz}, 3 \mathrm{H}) ;{ }^{13} \mathrm{C} \mathrm{NMR}\left(100 \mathrm{MHz}, \mathrm{CDCl}_{3}, \mathrm{ppm}\right): \delta$ $144.8,141.2,140.0,128.8,127.3,127.2,127.1,62.3,53.3,34.5,34.0,25.5,23.3$. Liu, Y.; Du, H. J. Am. Chem. Soc. 2013, 135, 12968. 


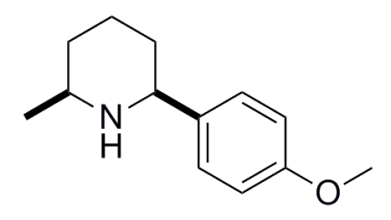

Cis-2-(4-methoxyphenyl)-6-methylpiperidine (5l): $0.0460 \mathrm{~g}, 56 \%$ yield, cis/trans $=$ 99/1, colorless oil; ${ }^{1} \mathrm{H}$ NMR (400 MHz, $\left.\mathrm{CDCl}_{3}, \mathrm{ppm}\right): \delta 7.29$ (d, $\left.J=8.4 \mathrm{~Hz}, 2 \mathrm{H}\right), 6.85$ $(\mathrm{d}, J=8.4 \mathrm{~Hz}, 2 \mathrm{H}), 3.79(\mathrm{~s}, 3 \mathrm{H}), 3.67-3.57(\mathrm{~m}, 1 \mathrm{H}), 2.91-2.69(\mathrm{~m}, 1 \mathrm{H}), 1.92-1.81(\mathrm{~m}$, $1 \mathrm{H}), 1.79-1.56(\mathrm{~m}, 3 \mathrm{H}), 1.56-1.34(\mathrm{~m}, 2 \mathrm{H}), 1.30-1.10(\mathrm{~m}, 1 \mathrm{H}), 1.10(\mathrm{~d}, J=6.4 \mathrm{~Hz}$ $3 \mathrm{H}) ;{ }^{13} \mathrm{C} \mathrm{NMR}\left(100 \mathrm{MHz}, \mathrm{CDCl}_{3}, \mathrm{ppm}\right): \delta 158.8,138.0,128.0,113.9,62.0,55.5$, $53.4,34.4,34.0,25.6,23.3$.

Liu, Y.; Du, H. J. Am. Chem. Soc. 2013, 135, 12968.<smiles>CC1CCCC(c2ccc(C(F)(F)F)cc2)N1</smiles>

Cis-2-methyl-6-[4-(trifluoromethyl)phenyl]piperidine (5m): 0.0797 g, 82\% yield, cis/trans $=86 / 14$, colorless oil; ${ }^{1} \mathrm{H}$ NMR $\left(400 \mathrm{MHz}, \mathrm{CDCl}_{3}, \mathrm{ppm}\right): \delta 7.56(\mathrm{~d}, J=8.4$ $\mathrm{Hz}, 2 \mathrm{H}), 7.49(\mathrm{~d}, J=8.4 \mathrm{~Hz}, 2 \mathrm{H}), 3.78-3.66(\mathrm{~m}, 1 \mathrm{H}), 2.89-2.73(\mathrm{~m}, 1 \mathrm{H}), 1.93-1.83(\mathrm{~m}$, $1 \mathrm{H}), 1.81-1.70(\mathrm{~m}, 1 \mathrm{H}), 1.70-1.61(\mathrm{~m}, 1 \mathrm{H}), 1.60-1.33(\mathrm{~m}, 3 \mathrm{H}), 1.31-1.12(\mathrm{~m}, 1 \mathrm{H})$, $1.12\left(\mathrm{~d}, J_{C-F}=6.2 \mathrm{~Hz}, 3 \mathrm{H}\right) ;{ }^{13} \mathrm{C} \mathrm{NMR}\left(100 \mathrm{MHz}, \mathrm{CDCl}_{3}, \mathrm{ppm}\right): \delta 149.8,129.4\left(\mathrm{q}, J_{C-F}\right.$ $=32.0 \mathrm{~Hz}), 127.2,125.5\left(\mathrm{q}, J_{C-F}=4.0 \mathrm{~Hz}\right), 123.1,62.3,53.2,34.7,33.9,25.5,23.2$.

Liu, Y.; Du, H. J. Am. Chem. Soc. 2013, 135, 12968.<smiles>Cc1ccc(OCC2CCCC(COc3ccc(C)cc3)N2)cc1</smiles>

Cis-2,6-bis[(p-tolyloxy)methyl]piperidine (5n): 0.1044 g, 80\% yield, cis/trans = 
99/1, white solid; ${ }^{1} \mathrm{H}$ NMR (400 MHz, $\left.\mathrm{CDCl}_{3}, \mathrm{ppm}\right): \delta 7.06$ (d, $\left.J=8.4 \mathrm{~Hz}, 4 \mathrm{H}\right), 6.81$ (d, $J=8.4 \mathrm{~Hz}, 4 \mathrm{H}), 3.94-3.85(\mathrm{~m}, 2 \mathrm{H}), 3.84-3.75(\mathrm{~m}, 2 \mathrm{H}), 3.12-2.99(\mathrm{~m}, 2 \mathrm{H}), 2.64$ (brs, 1H), $2.27(\mathrm{~s}, 6 \mathrm{H}), 1.93-1.83(\mathrm{~m}, 1 \mathrm{H}), 1.77-1.63(\mathrm{~m}, 2 \mathrm{H}), 1.56-1.39(\mathrm{~m}, 1 \mathrm{H})$, 1.30-1.13 (m, 2H); ${ }^{13} \mathrm{C}$ NMR (100 MHz, $\left.\mathrm{CDCl}_{3}, \mathrm{ppm}\right): \delta$ 157.0, 130.2, 130.0, 114.7, 73.0, 55.6, 28.7, 24.0, 20.7 .

Liu, Y.; Du, H. J. Am. Chem. Soc. 2013, 135, 12968.<smiles>c1ccc2cc(C3CCCCN3)ccc2c1</smiles>

2-(Naphthalen-2-yl)piperidine (5o): 0.0372 g, 44\% yield, white solid, m.p. $42-44{ }^{\circ} \mathrm{C}$; IR (film): 3321, 1633, 1601, 1440, 1316, $1107 \mathrm{~cm}^{-1} ;{ }^{1} \mathrm{H}$ NMR $\left(400 \mathrm{MHz}, \mathrm{CDCl}_{3}\right.$, ppm): $\delta$ 7.90-7.75 (m, 4H), 7.53-7.48 (m, 1H), 7.48-7.42 (m, 2H) 3.83-3.69 (m, 1H), 3.33-3.20 (m, 1H), 2.93-2.79 (m, 1H), 2.00-1.82 (m, 3H), 1.78-1.68 (m, 1H), 1.70-1.50 (m, 3H); ${ }^{13} \mathrm{C}$ NMR (100 MHz, $\left.\mathrm{CDCl}_{3}, \mathrm{ppm}\right): \delta$ 143.3, 133.8, 133.0, 128.2, $128.1,127.8,126.1,125.7,124.9,62.6,48.1,35.3,26.2,25.7$.

Liu, Y.; Du, H. J. Am. Chem. Soc. 2013, 135, 12968.<smiles>CCOC(=O)[C@H]1CCCN[C@H]1c1ccccc1</smiles>

Ethyl 2-phenylpiperidine-3-carboxylate (5p): 0.0411 g, 44\% yield, cis/trans = 67/33, yellow oil; IR (film): 3359, 1724, 1655, 1633, $1175 \mathrm{~cm}^{-1} ;{ }^{1} \mathrm{H}$ NMR (400 MHz, $\left.\mathrm{CDCl}_{3}, \mathrm{ppm}\right): \delta$ 7.48-7.15 (m, 5H), 4.04-3.69 (m, 3H), 3.39-3.23 (m, 0.67H) (cis 
isomer), 3.23-3.13 (m, 0.33H) (trans isomer), 3.04-2.56 (m, 3H), 2.22-2.04 (m, 1H), 2.00-1.45 (m, 3H), 1.02-0.86 (m, 3H); ${ }^{13} \mathrm{C}$ NMR (100 MHz, $\left.\mathrm{CDCl}_{3}, \mathrm{ppm}\right): \delta 173.7$, $142.2,128.5,128.2,127.9,127.8,126.9,126.2,64.3,61.2,60.1,59.9,50.5,47.2,47.1$, 44.6, 28.9, 28.0, 25.1, 22.1, 14.1; HRMS (ESI) calcd. for $\mathrm{C}_{14} \mathrm{H}_{20} \mathrm{NO}_{2}[\mathrm{M}+\mathrm{H}]^{+}$: 234.1494, Found: 234.1486.

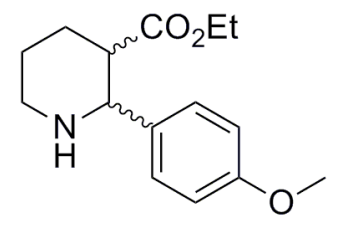

Ethyl 2-(4-methoxyphenyl)piperidine-3-carboxylate (5q): 0.0558 g, 53\% yield, cis/trans $=70 / 30$, yellow oil; IR (film): 3322, 1720, 1514, 1247, $1177 \mathrm{~cm}^{-1} ;{ }^{1} \mathrm{H}$ NMR (400 MHz, $\left.\mathrm{CDCl}_{3}, \mathrm{ppm}\right): \delta$ 7.39-7.10 (m, 2H), 6.96-6.74 (m, 2H), 3.98-3.82 (m, 3H), $3.77(\mathrm{~s}, 3 \mathrm{H}), 3.40-3.21(\mathrm{~m}, 0.7 \mathrm{H})$ (cis isomer), 3.21-3.09 (m, 0.3H) (trans isomer), 2.99-2.55 (m, 3H), 2.26-1.60 (m, 4H), 1.59-1.43 (m, 1H), 1.12-0.90 (m, 3H); ${ }^{13} \mathrm{C}$ NMR (100 MHz, $\left.\mathrm{CDCl}_{3}, \mathrm{ppm}\right): \delta 173.8,158.5,134.4,128.9,127.3,113.8,113.6$, $63.5,60.7,60.1,59.9,55.4,50.5,47.2,47.1,44.6,29.0,27.8,25.0,22.1,14.2$; HRMS (ESI) calcd. for $\mathrm{C}_{15} \mathrm{H}_{22} \mathrm{NO}_{3}[\mathrm{M}+\mathrm{H}]^{+}: 264.1600$, Found: 264.1594 . 

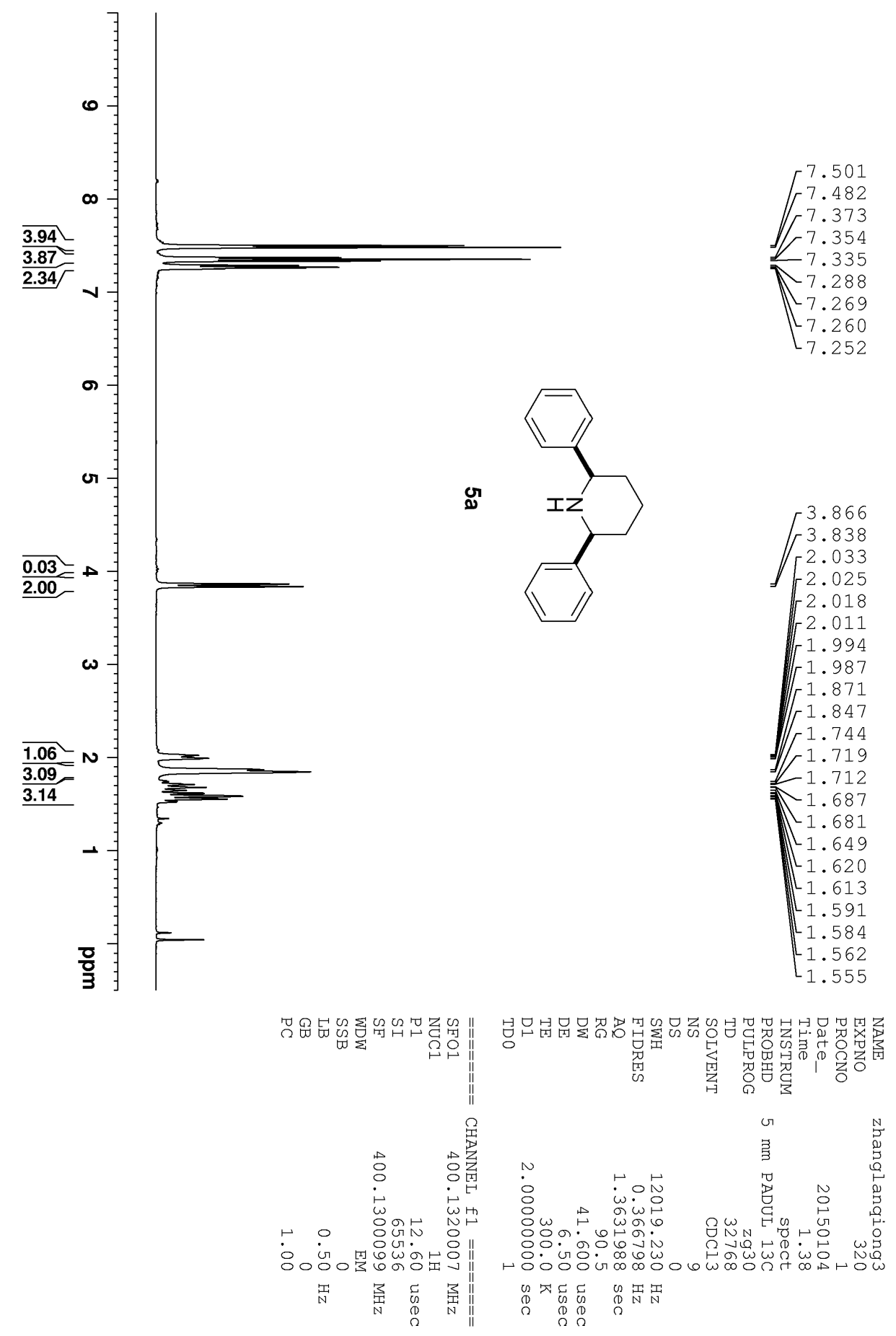


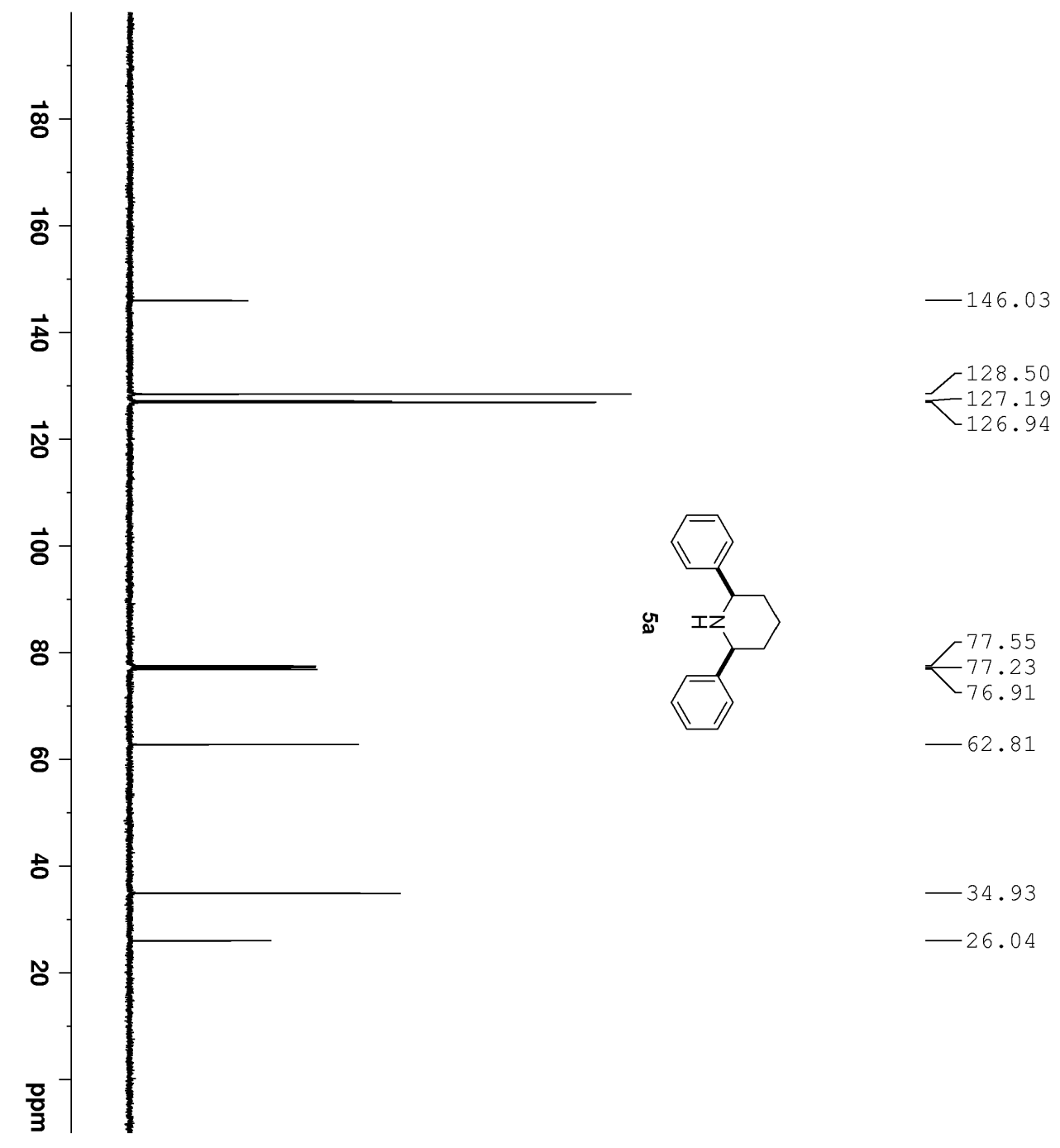

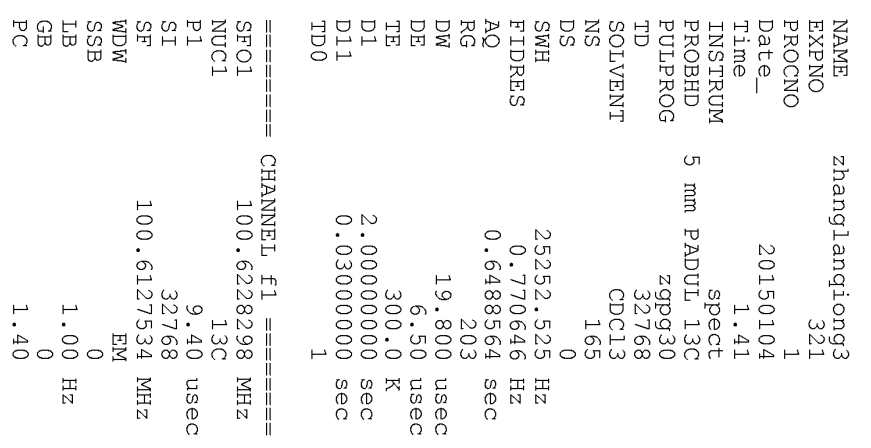




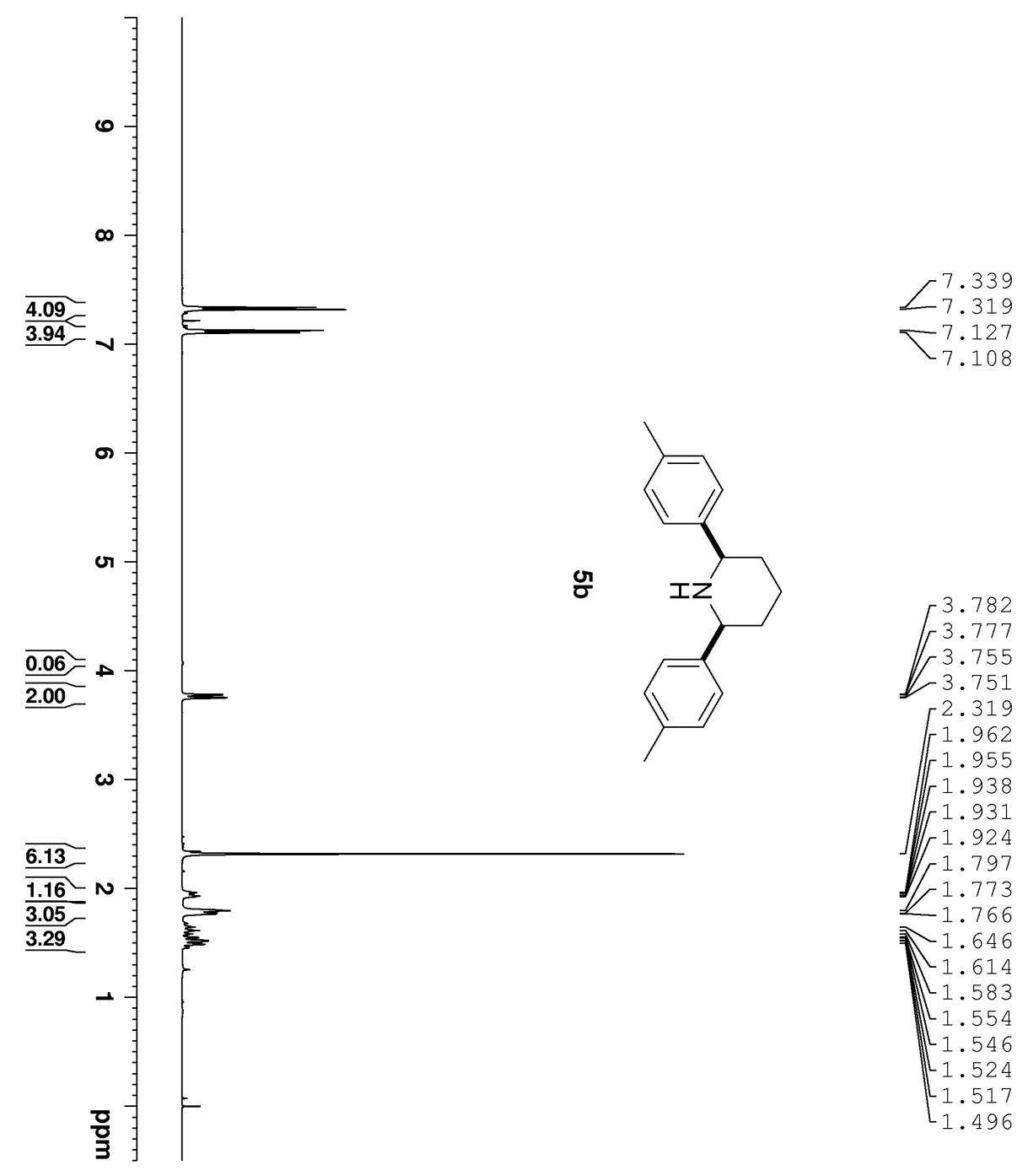

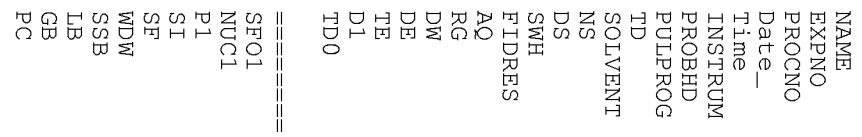

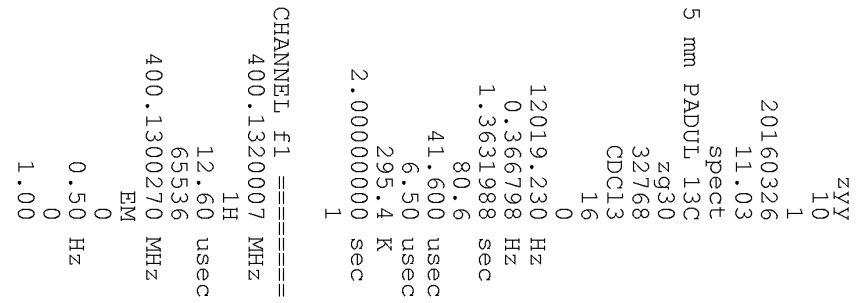




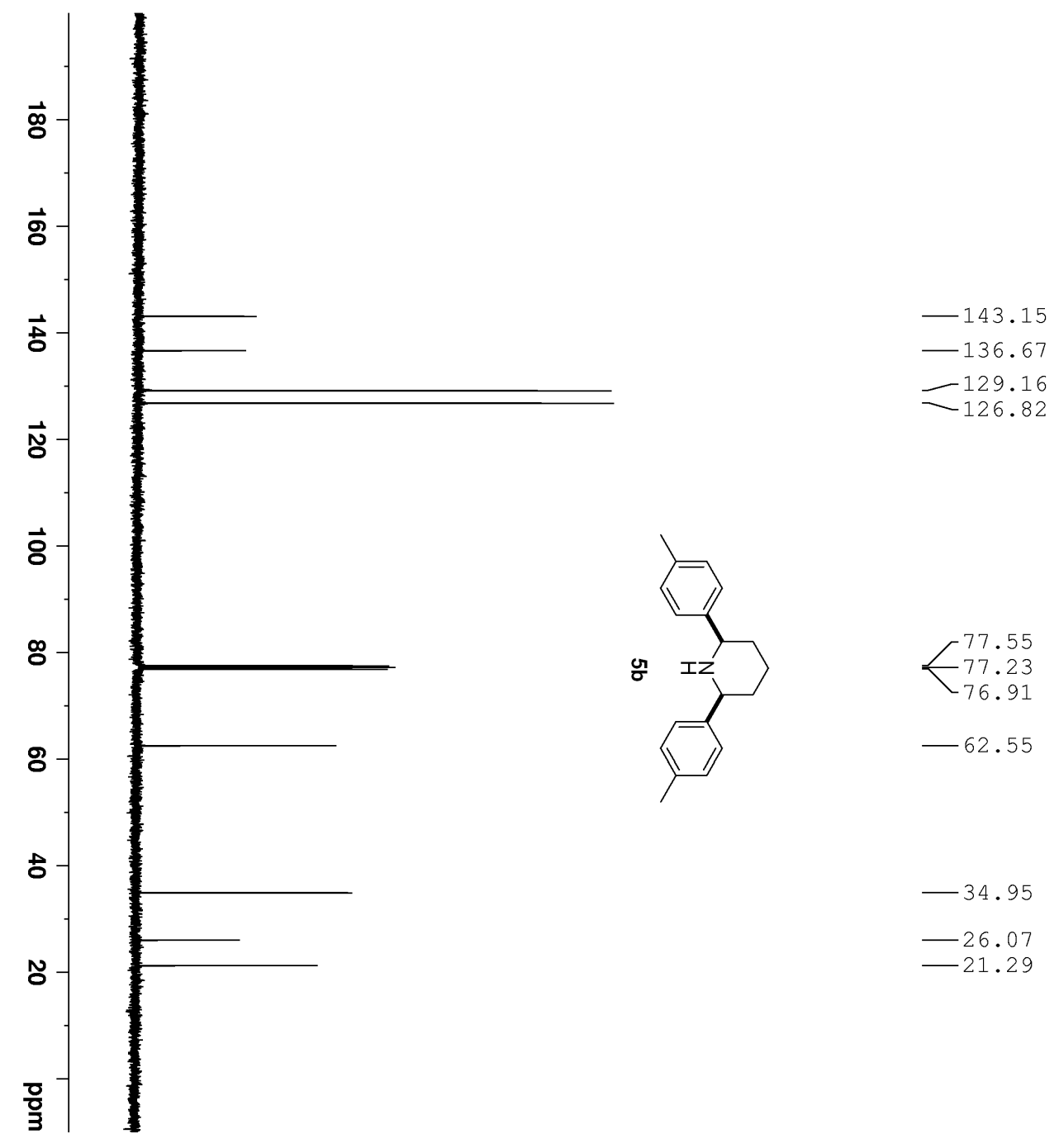

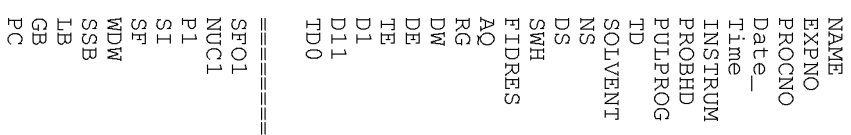

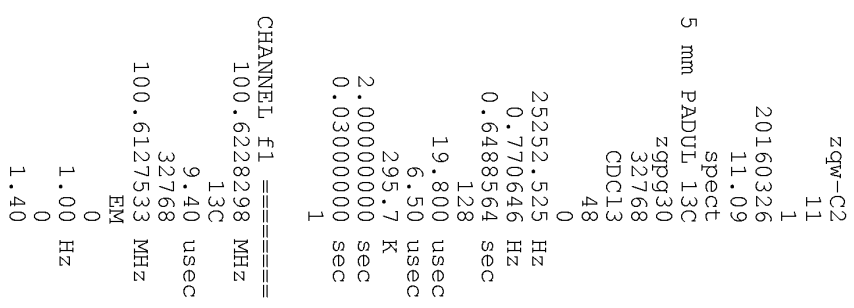




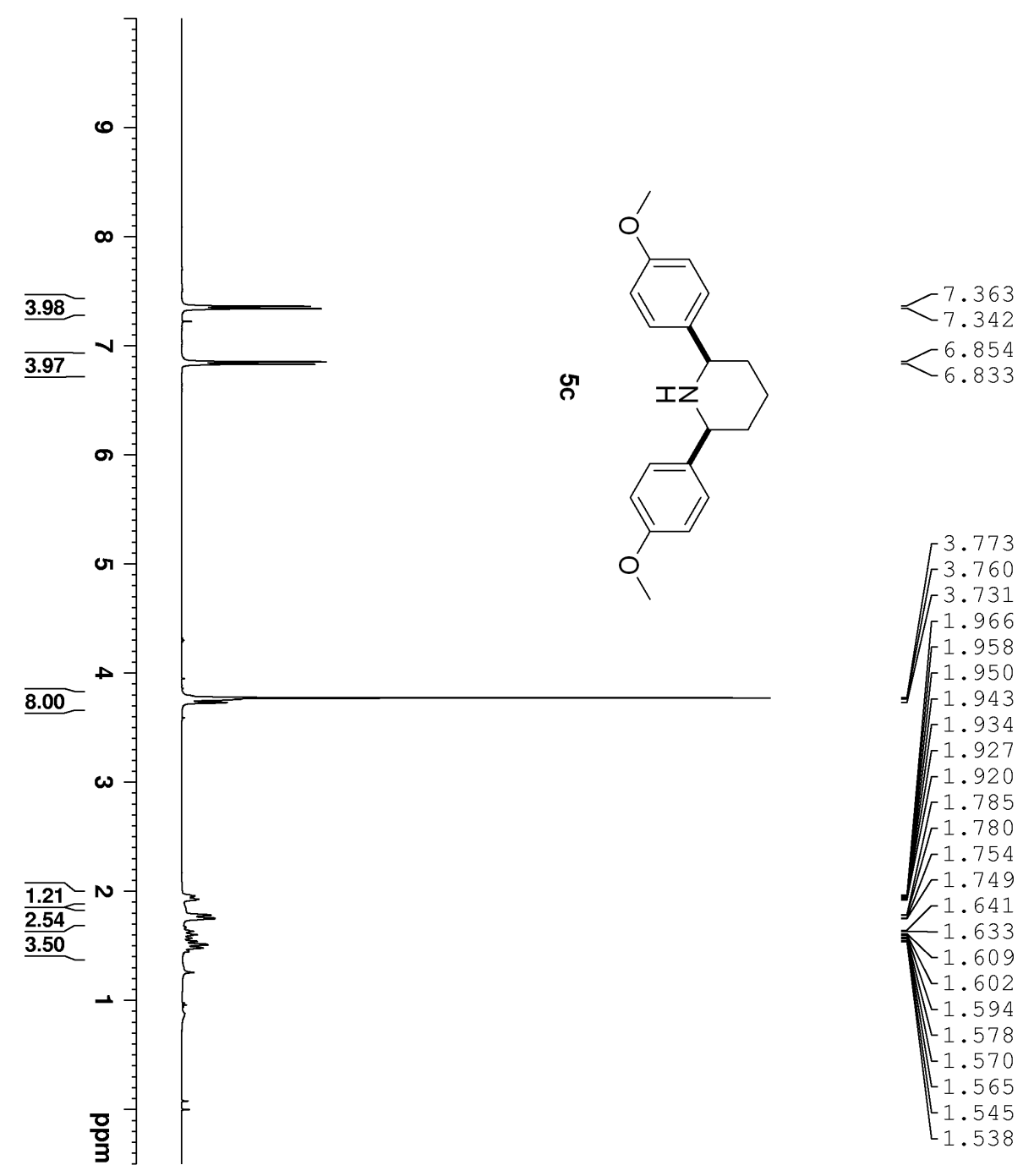

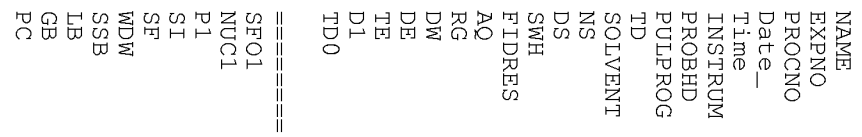

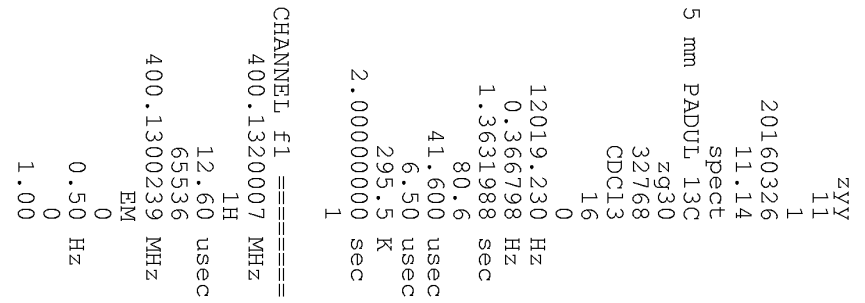




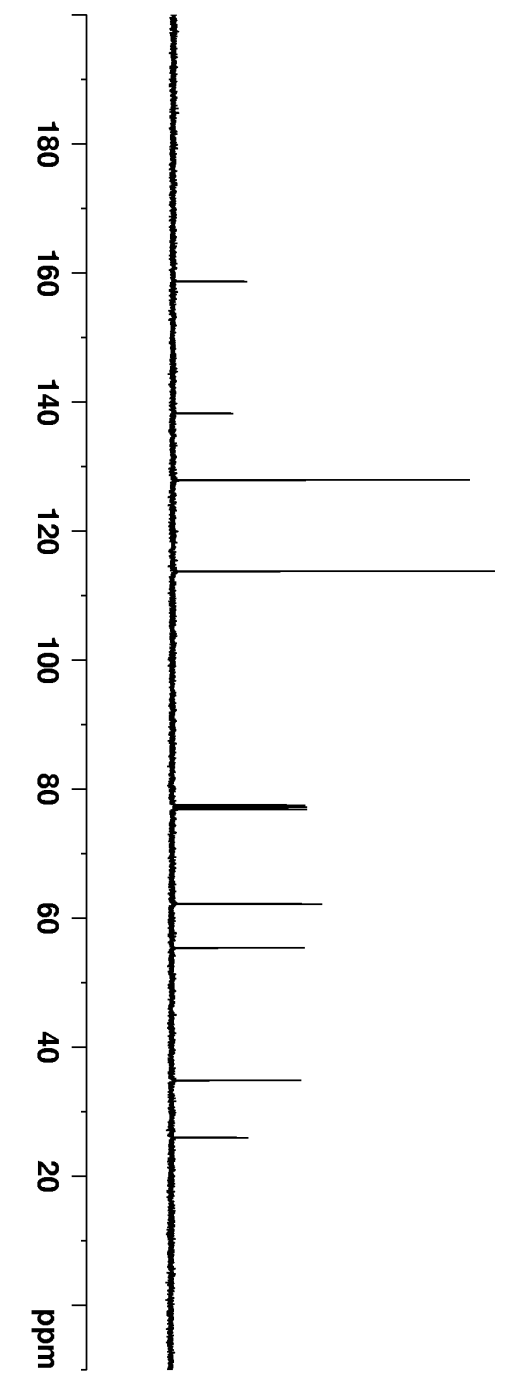

$-158.74$

$-138.28$

$-127.93$

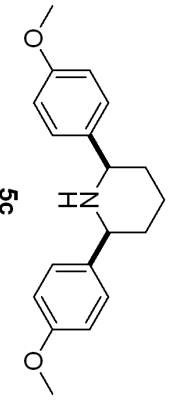

$-113.80$

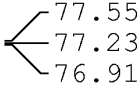

$-62.23$

$-55.42$

$-34.89$

$-26.00$

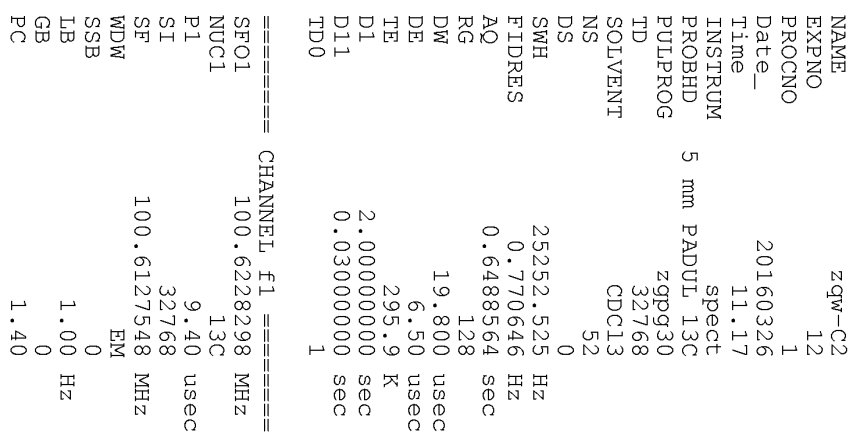




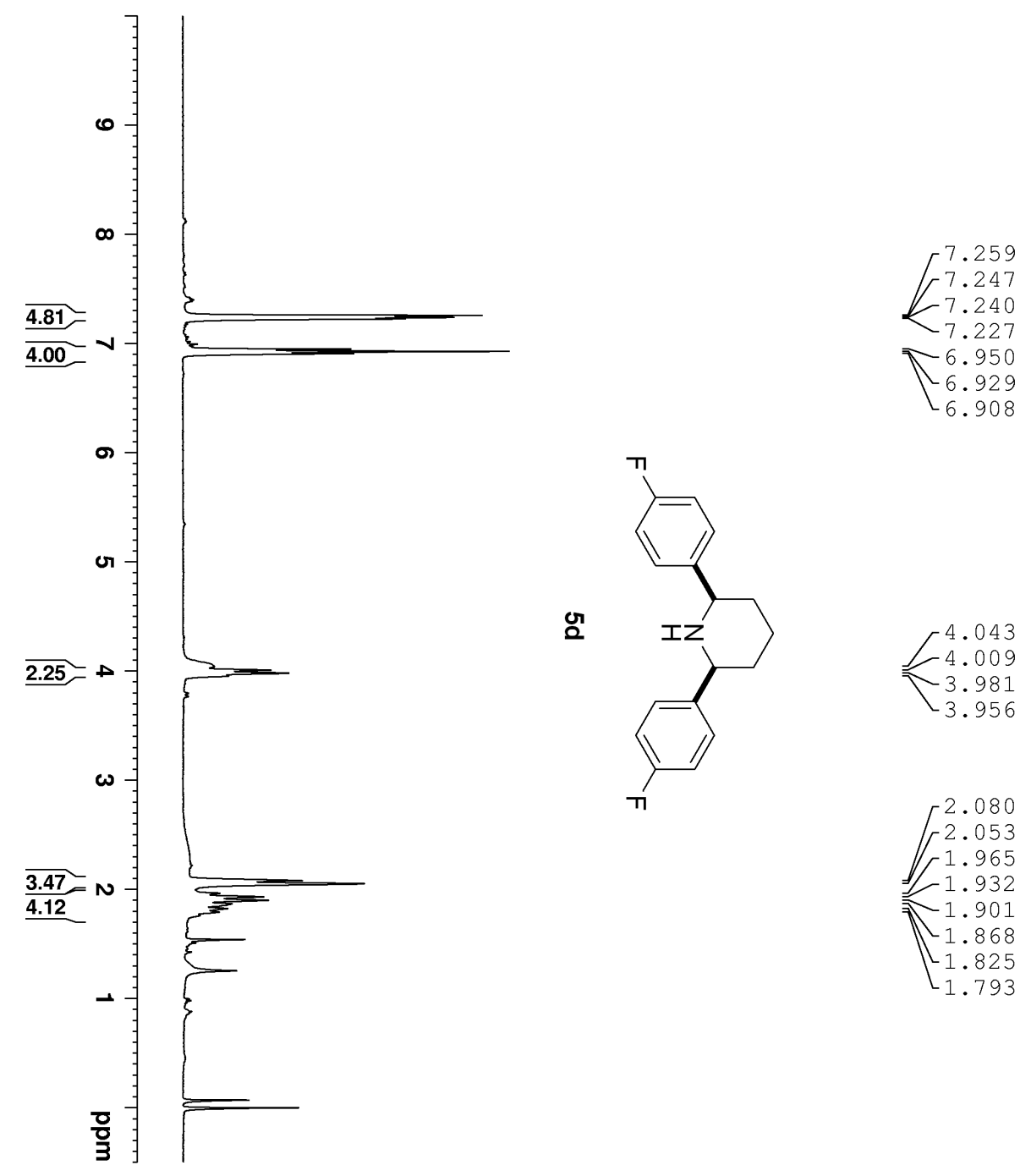

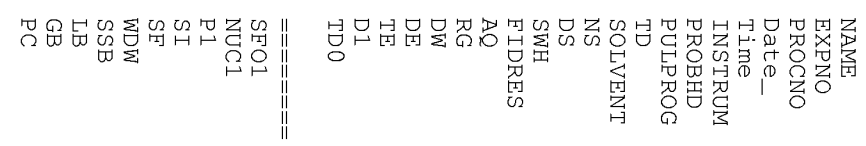

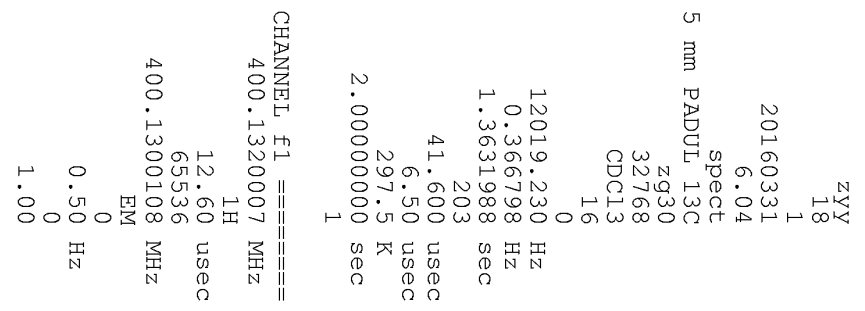




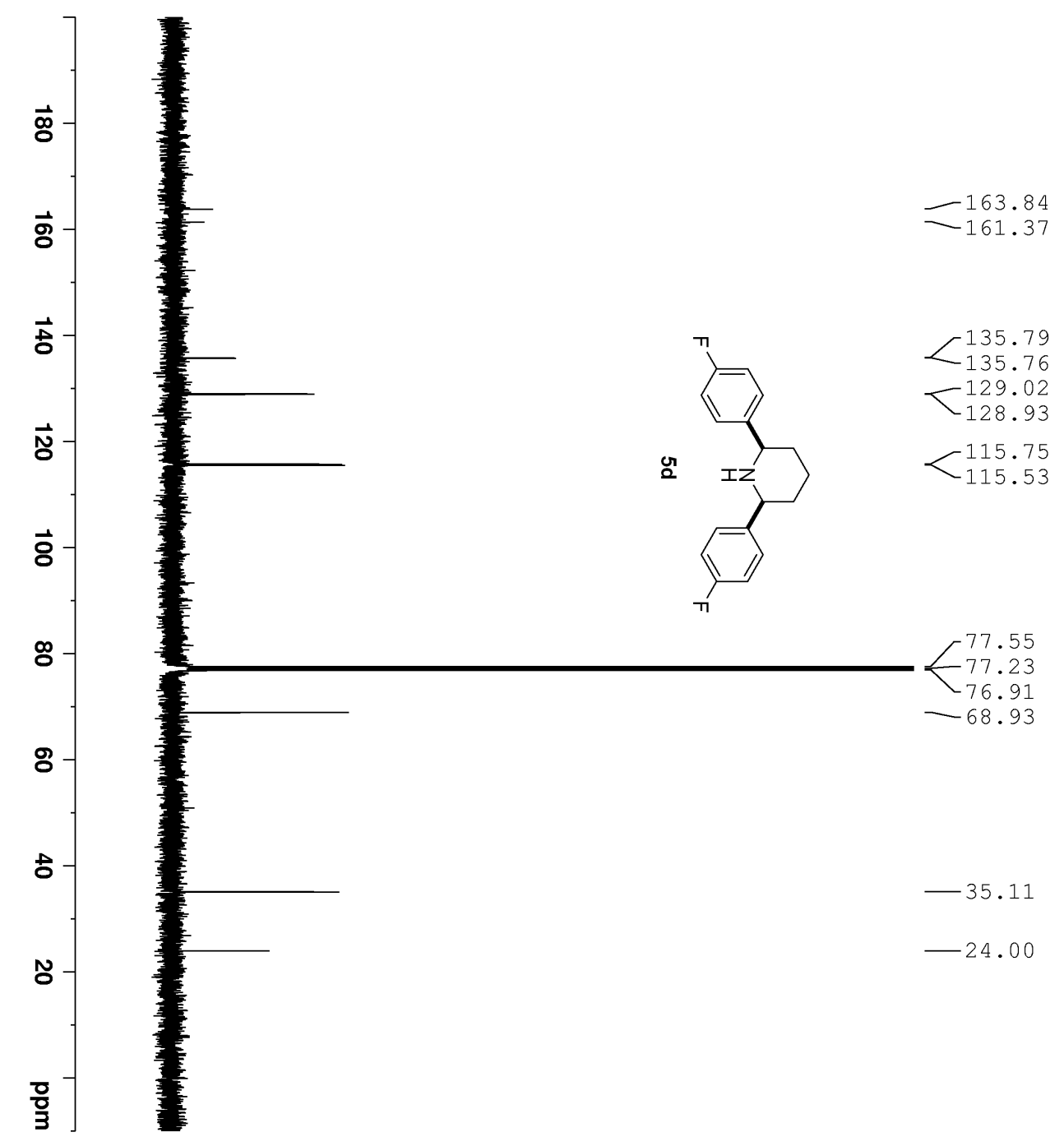

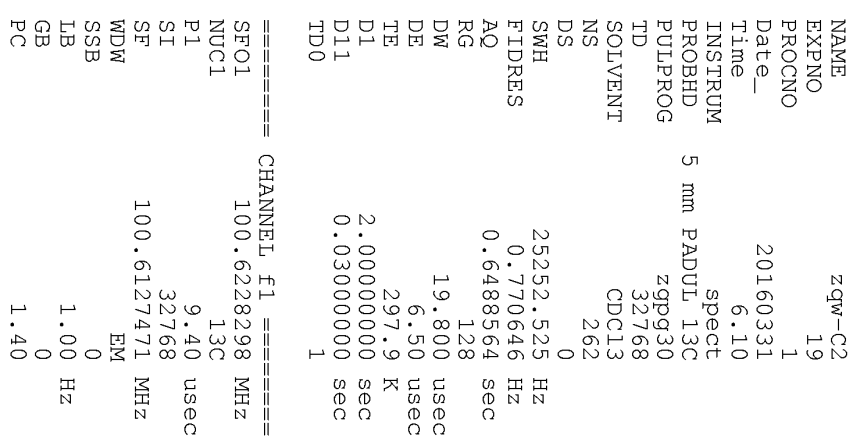




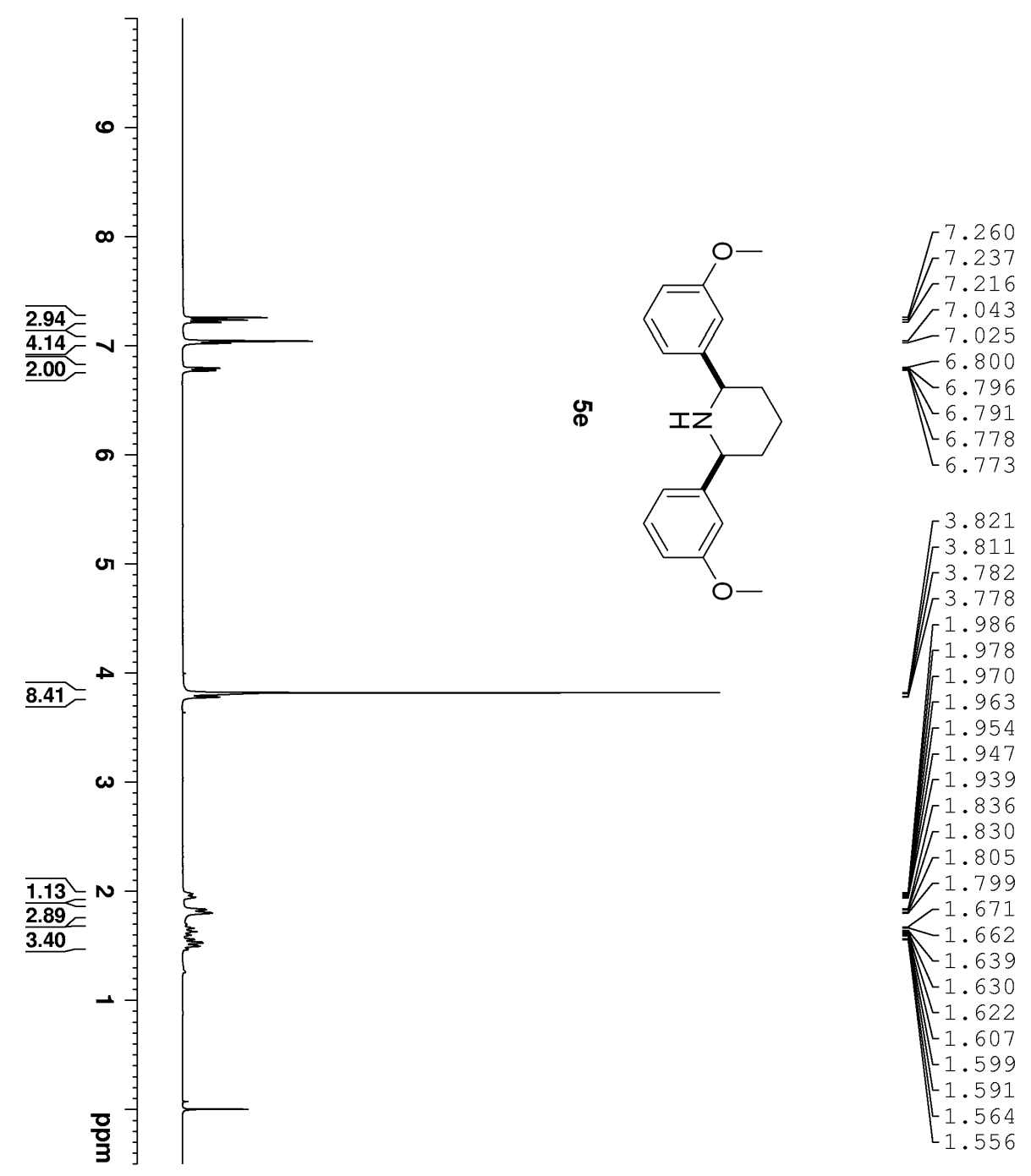

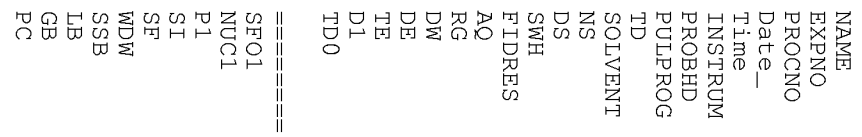

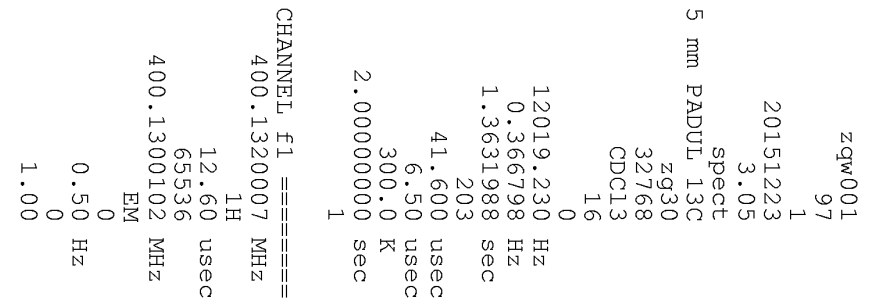




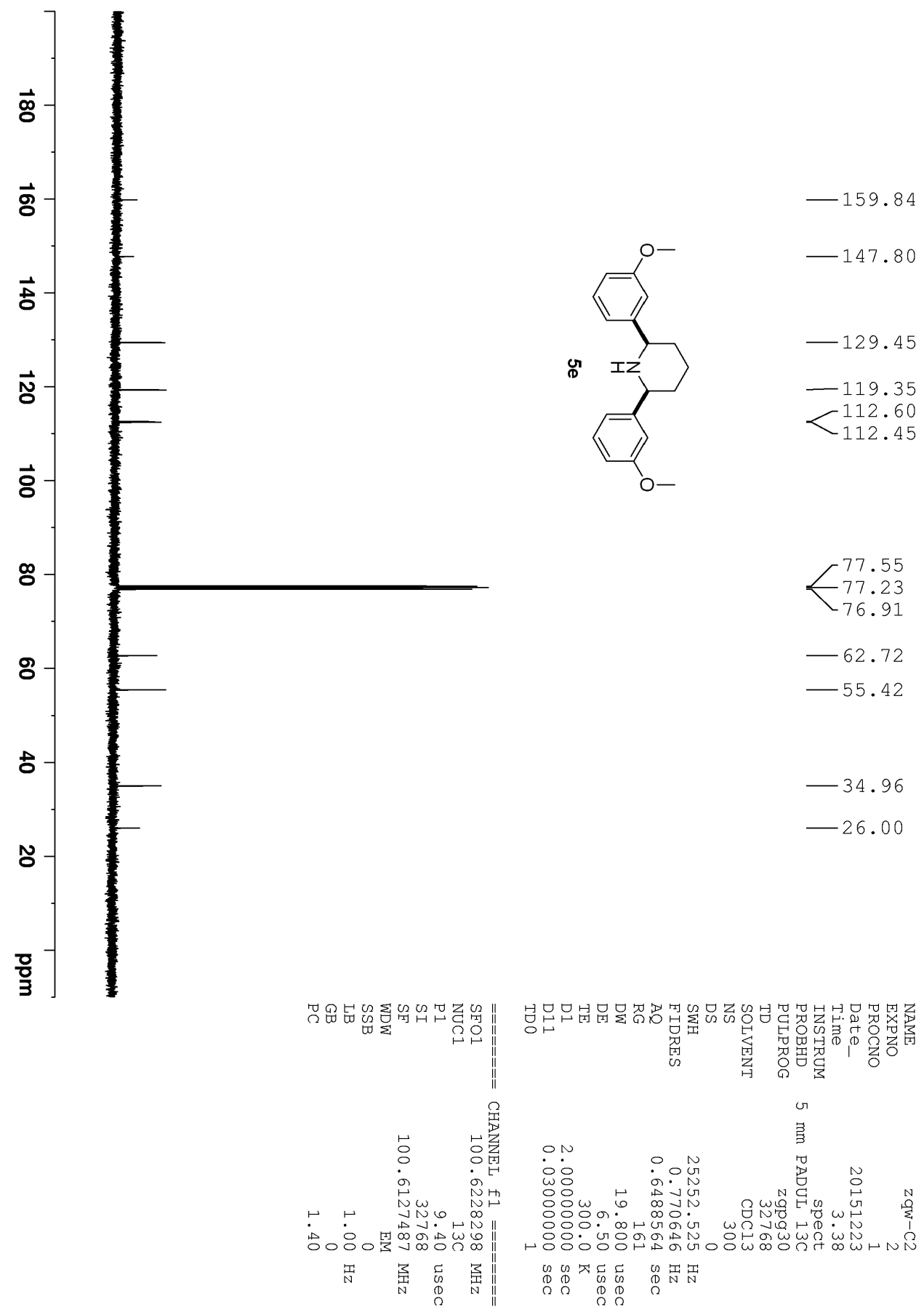




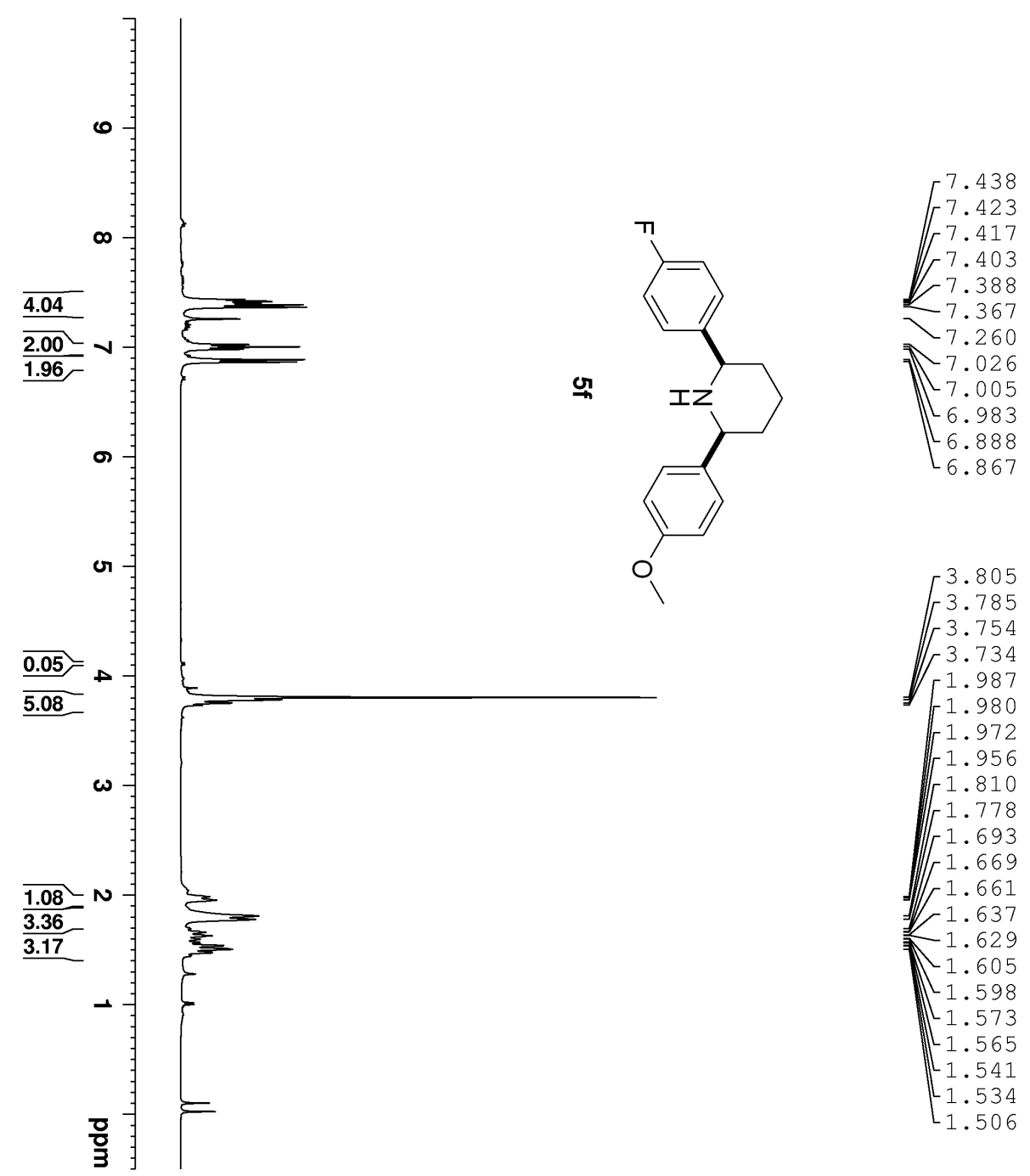

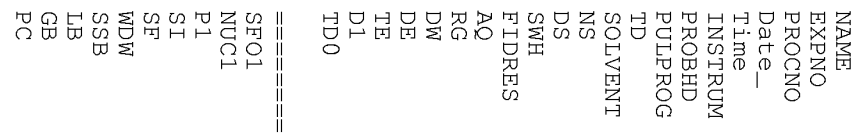

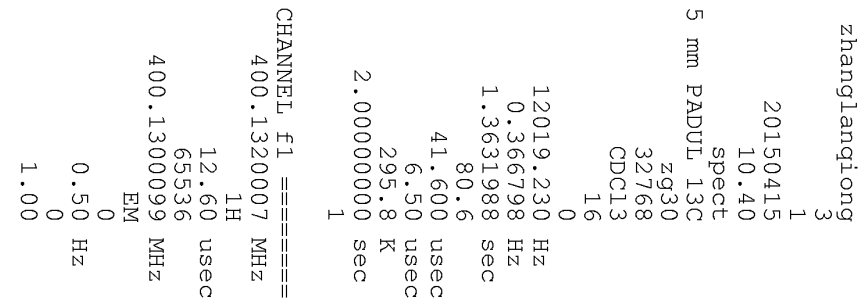



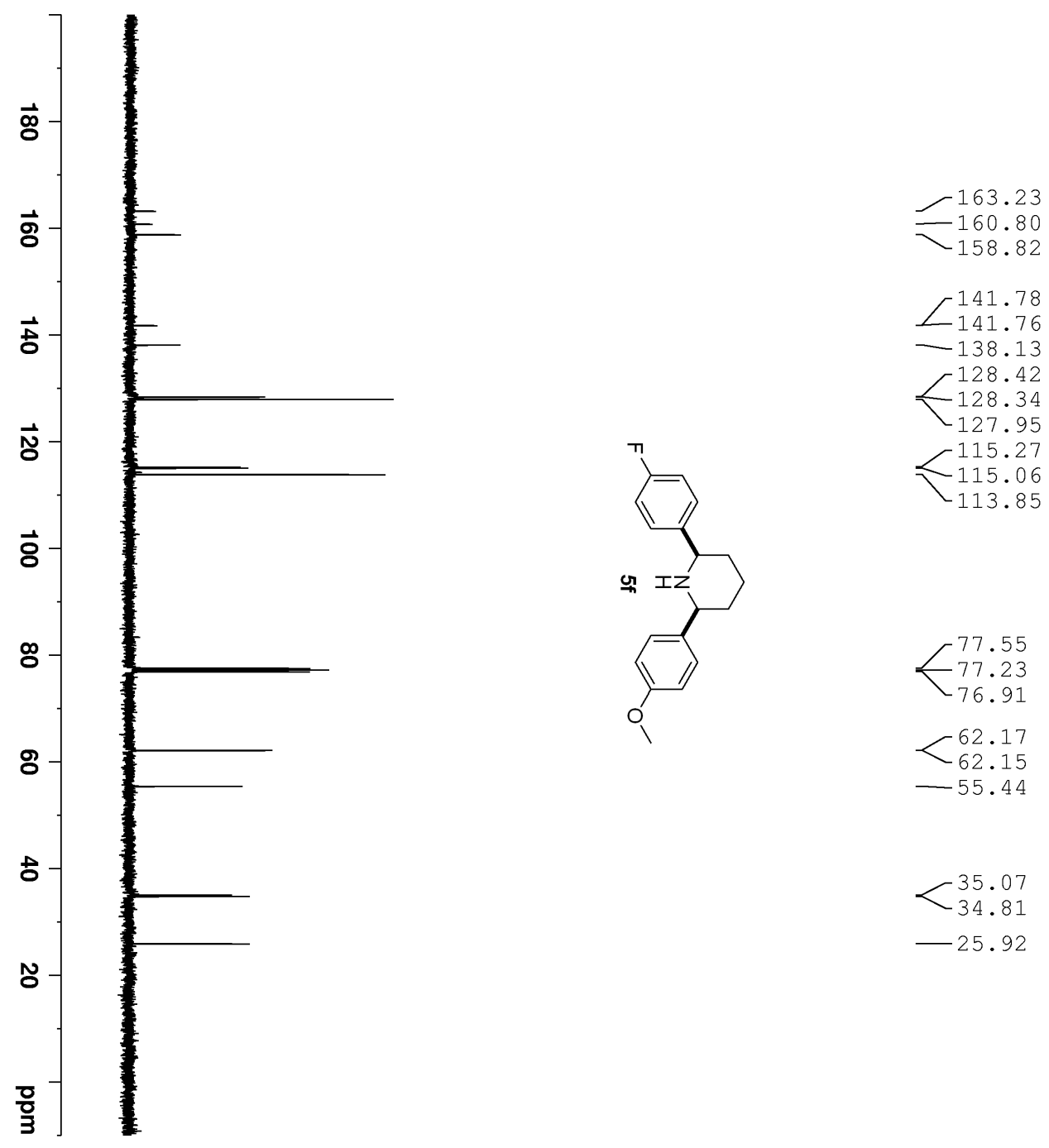

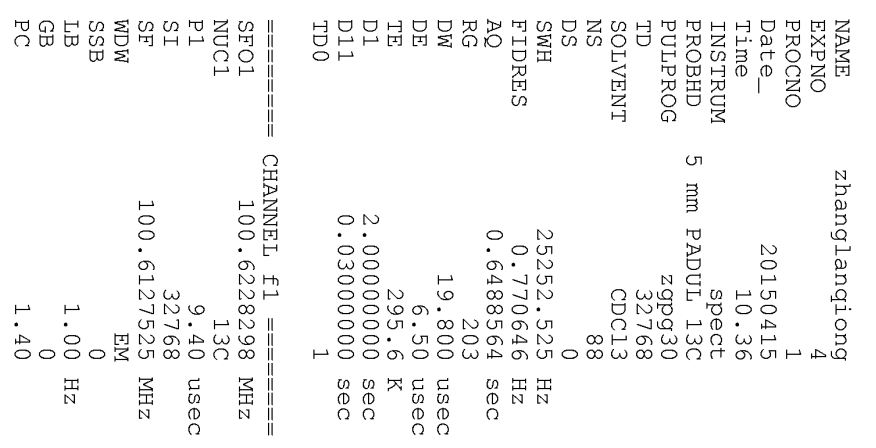




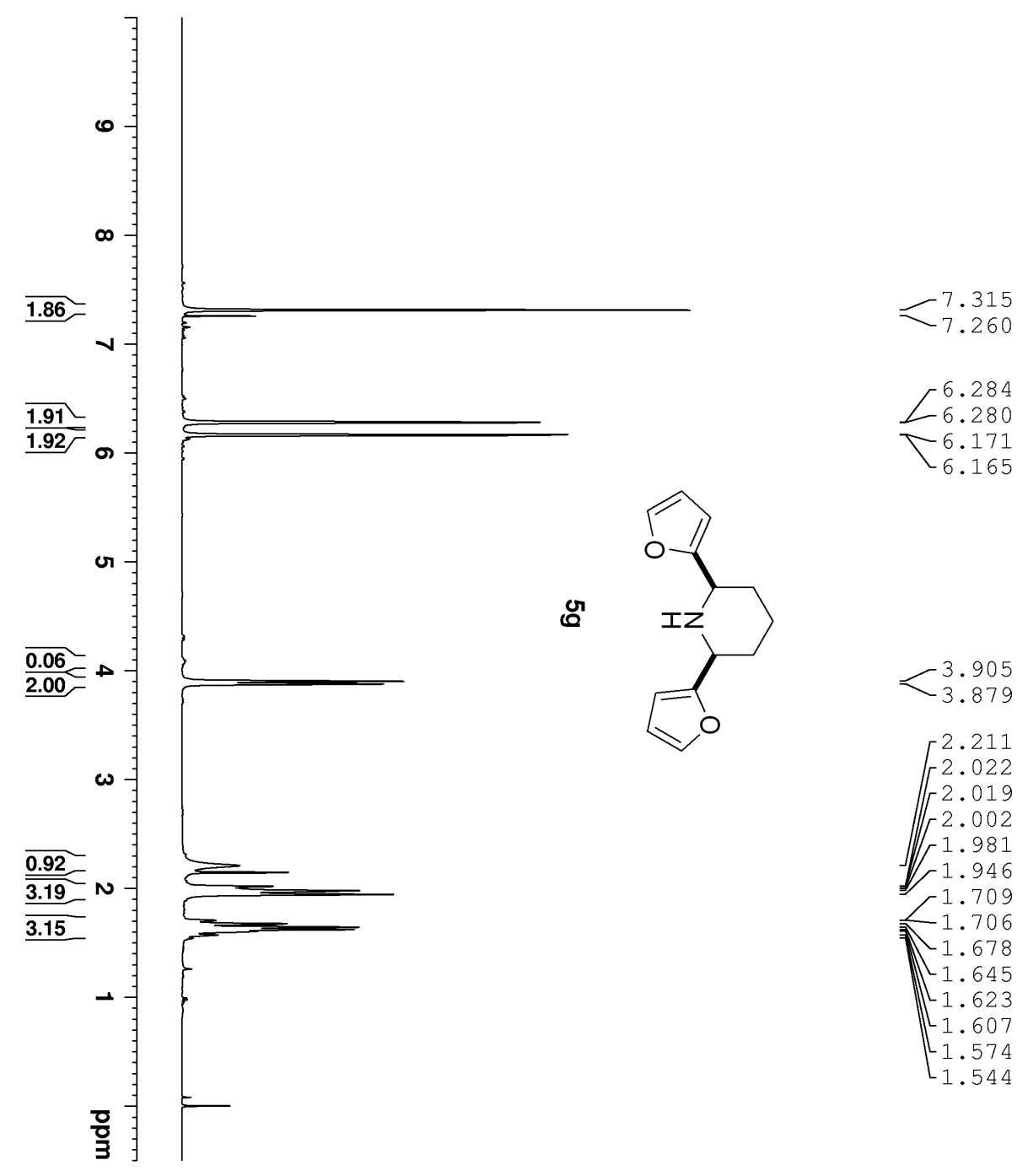

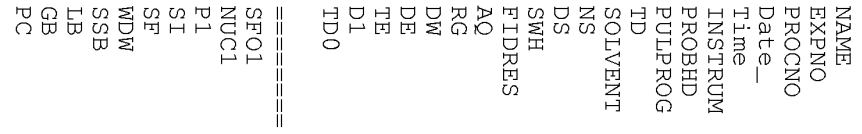

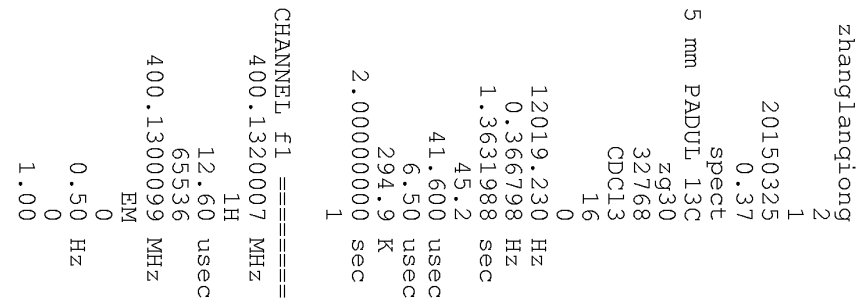




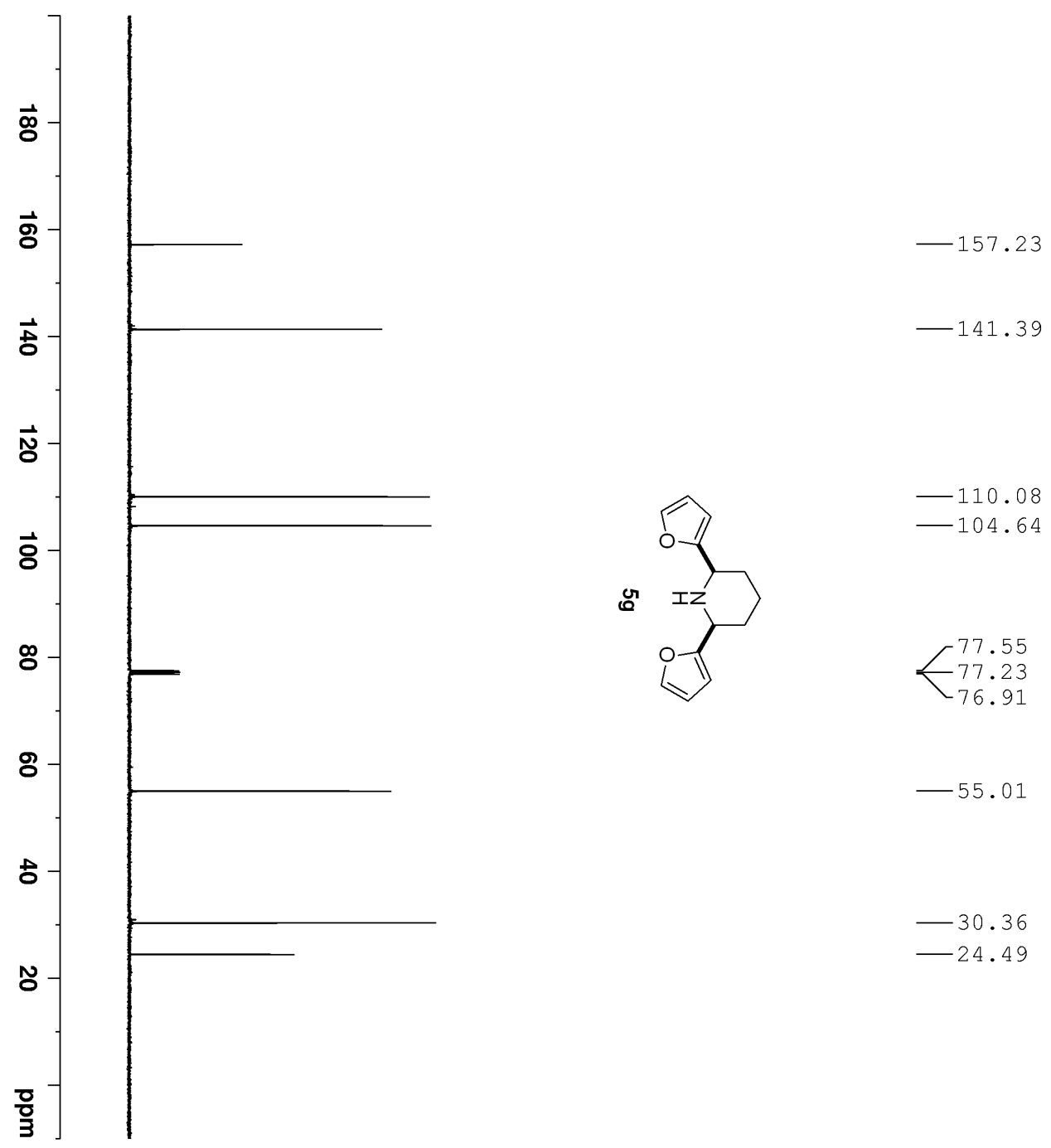

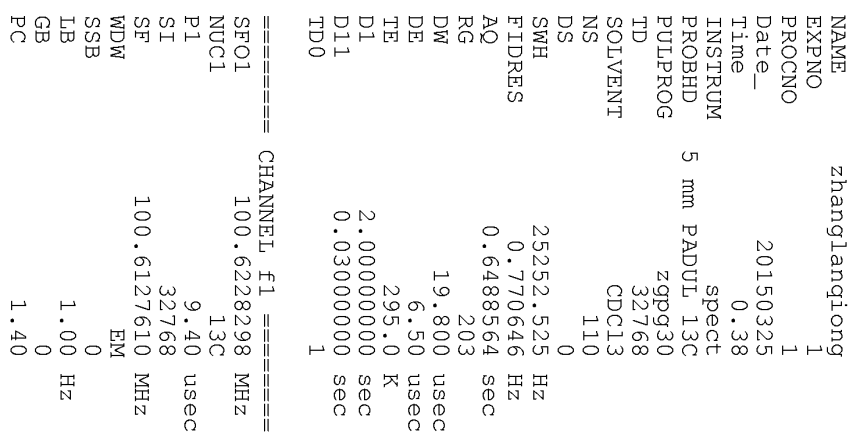




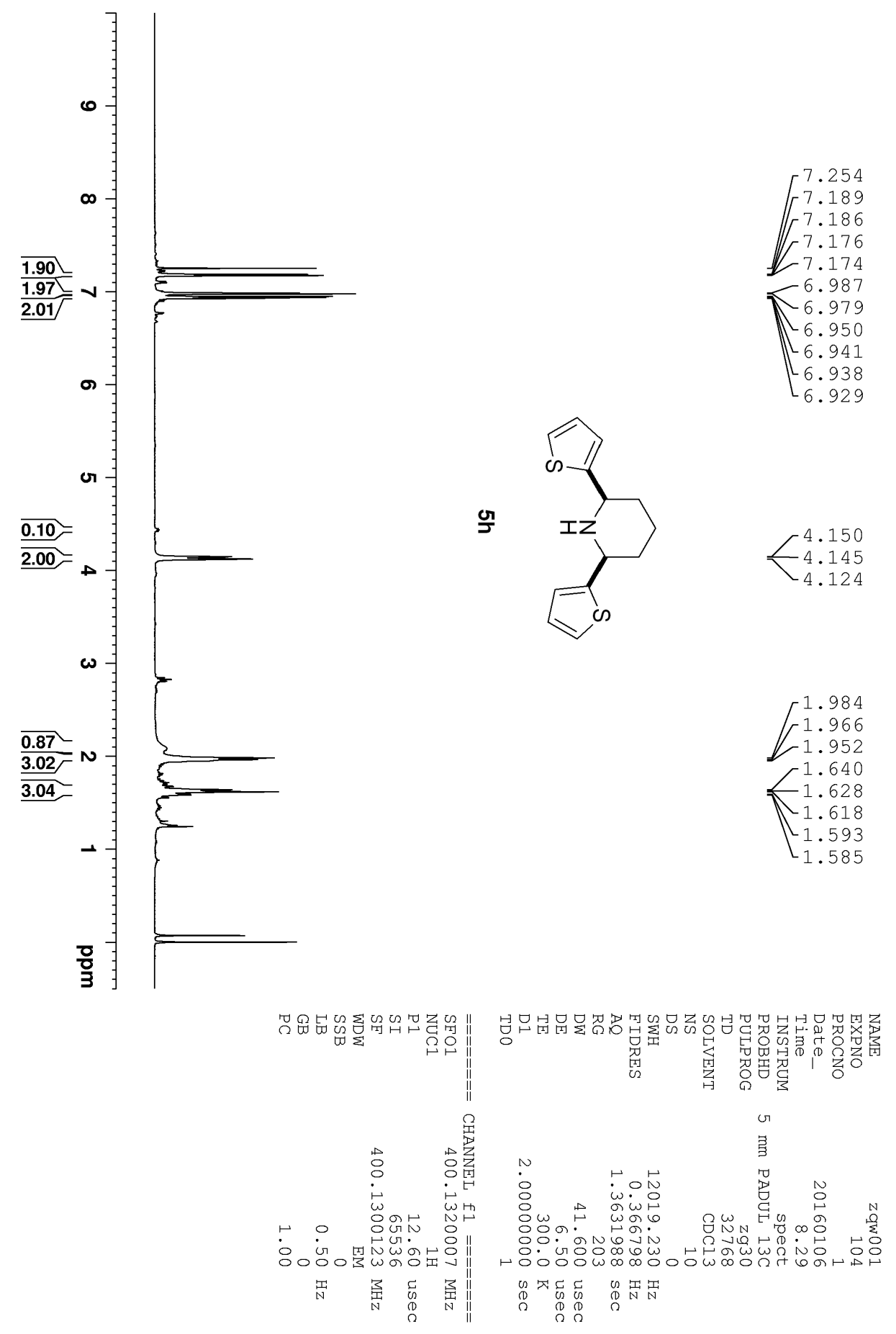



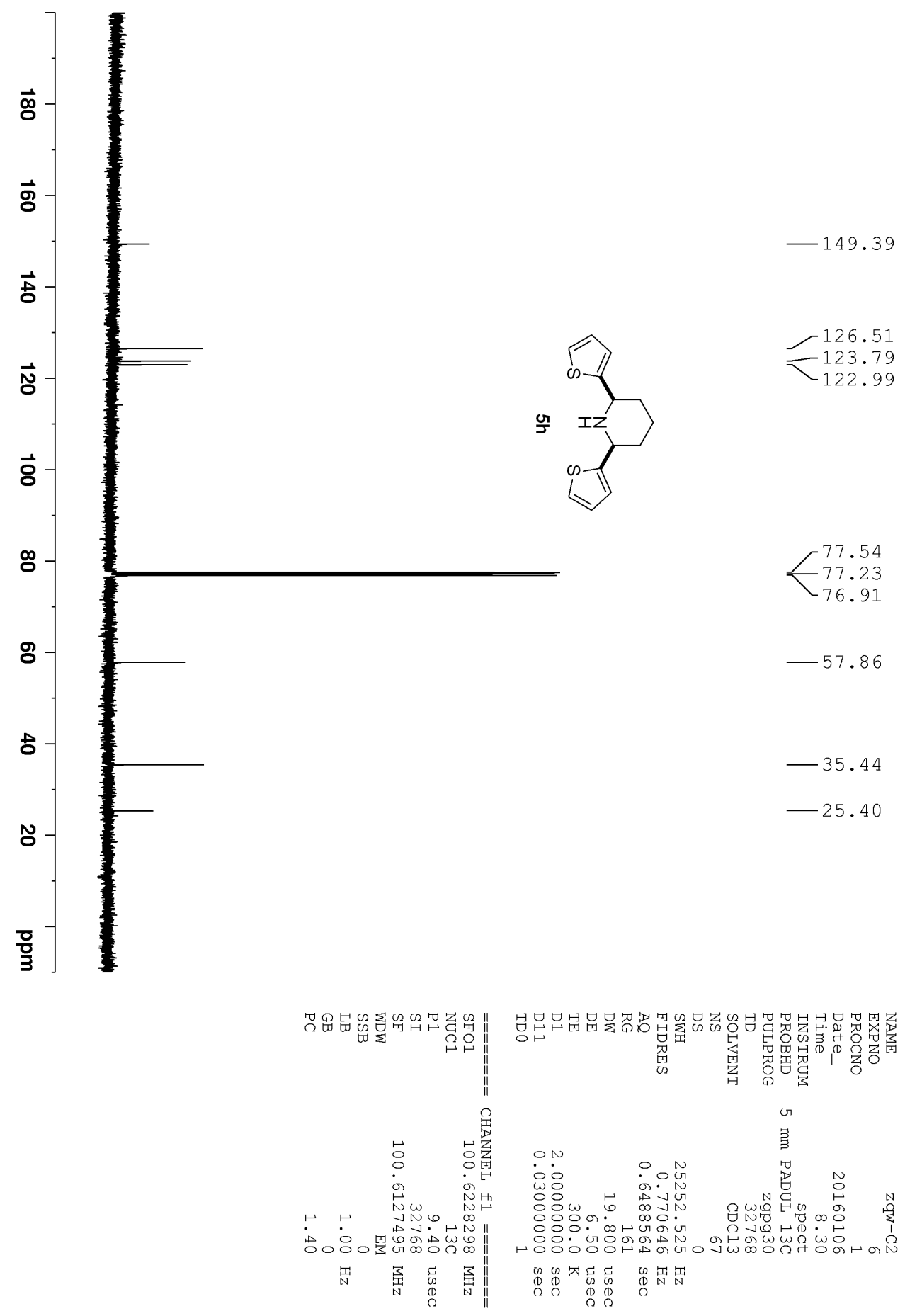


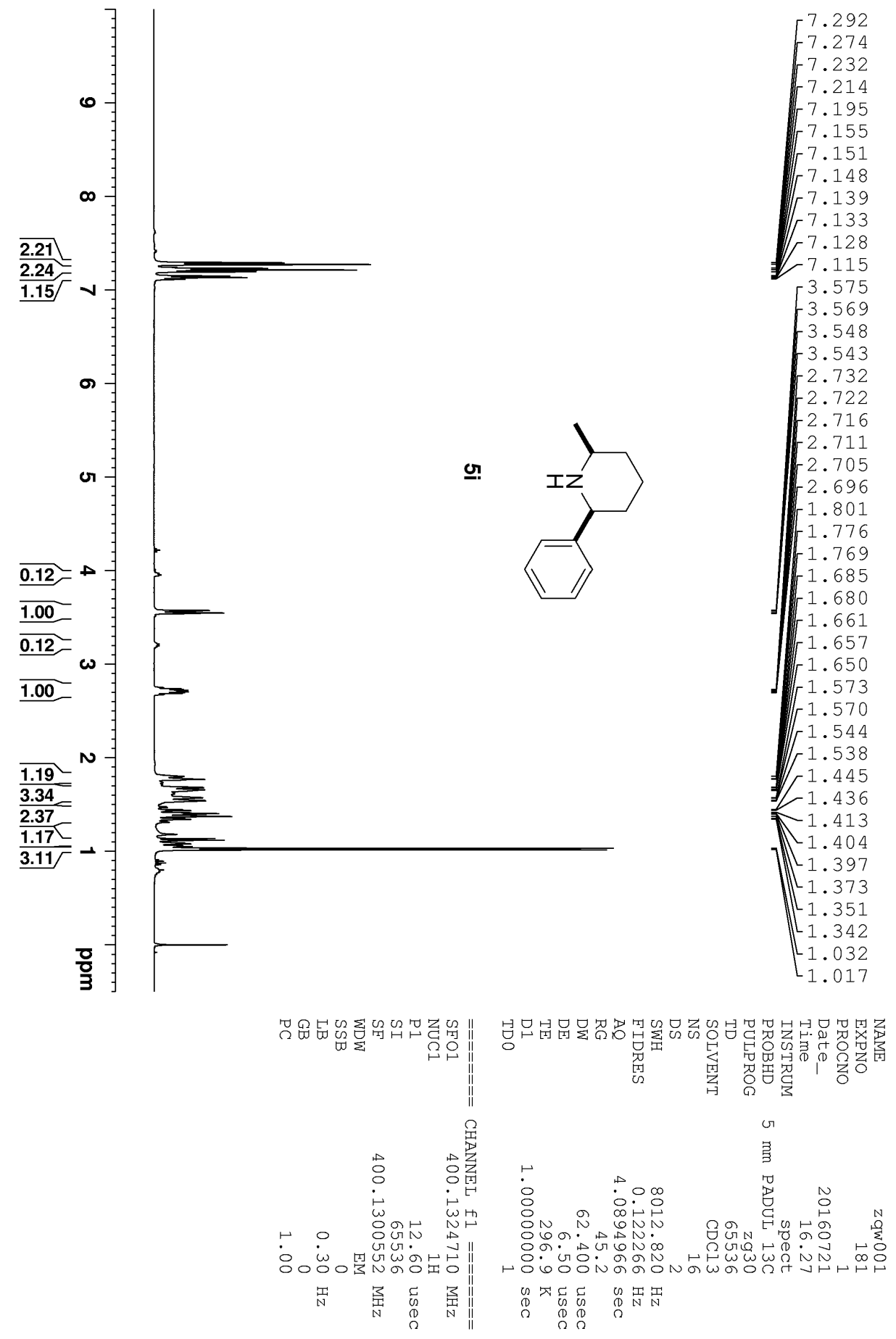




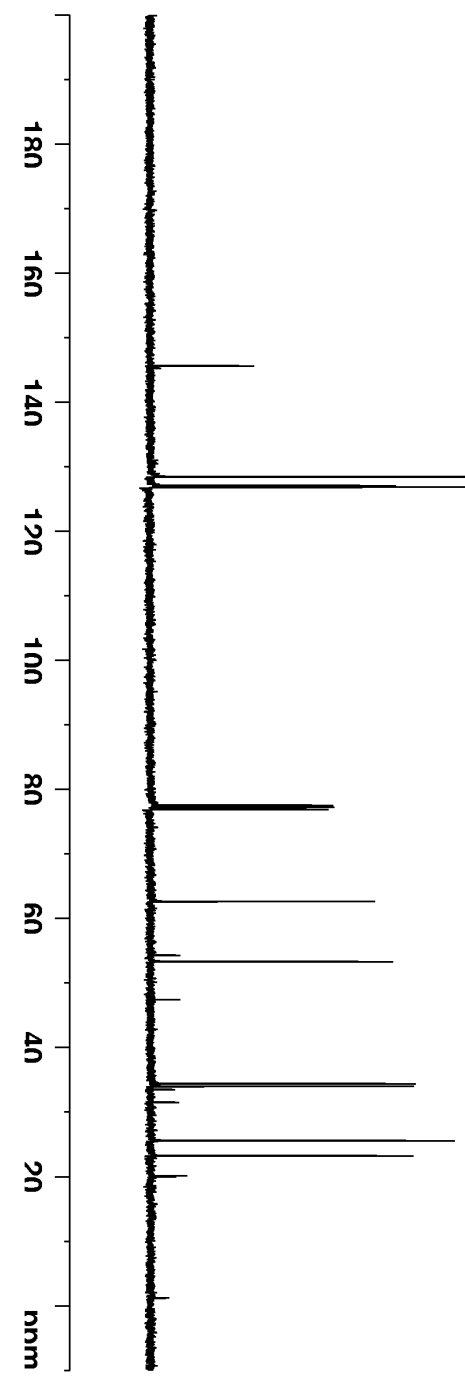

$-145.66$
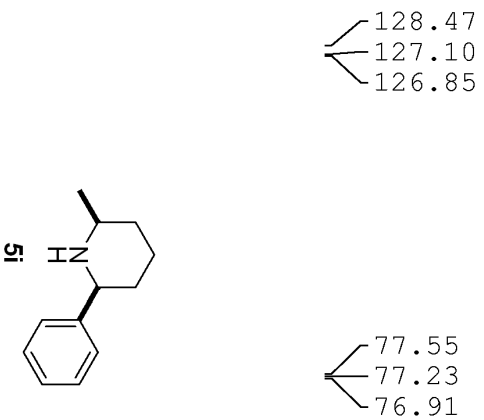

$-62.62$

$-53.32$

$<34.41$

$\begin{array}{r}33.99 \\ \hline\end{array}$

$-25.53$

$-23.22$

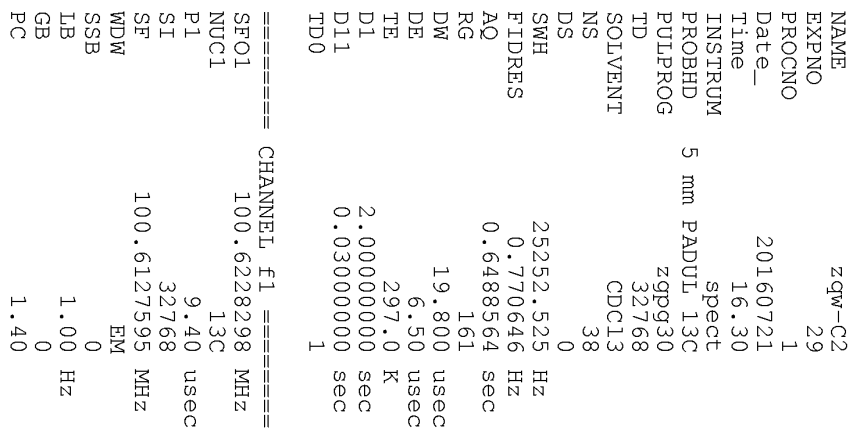




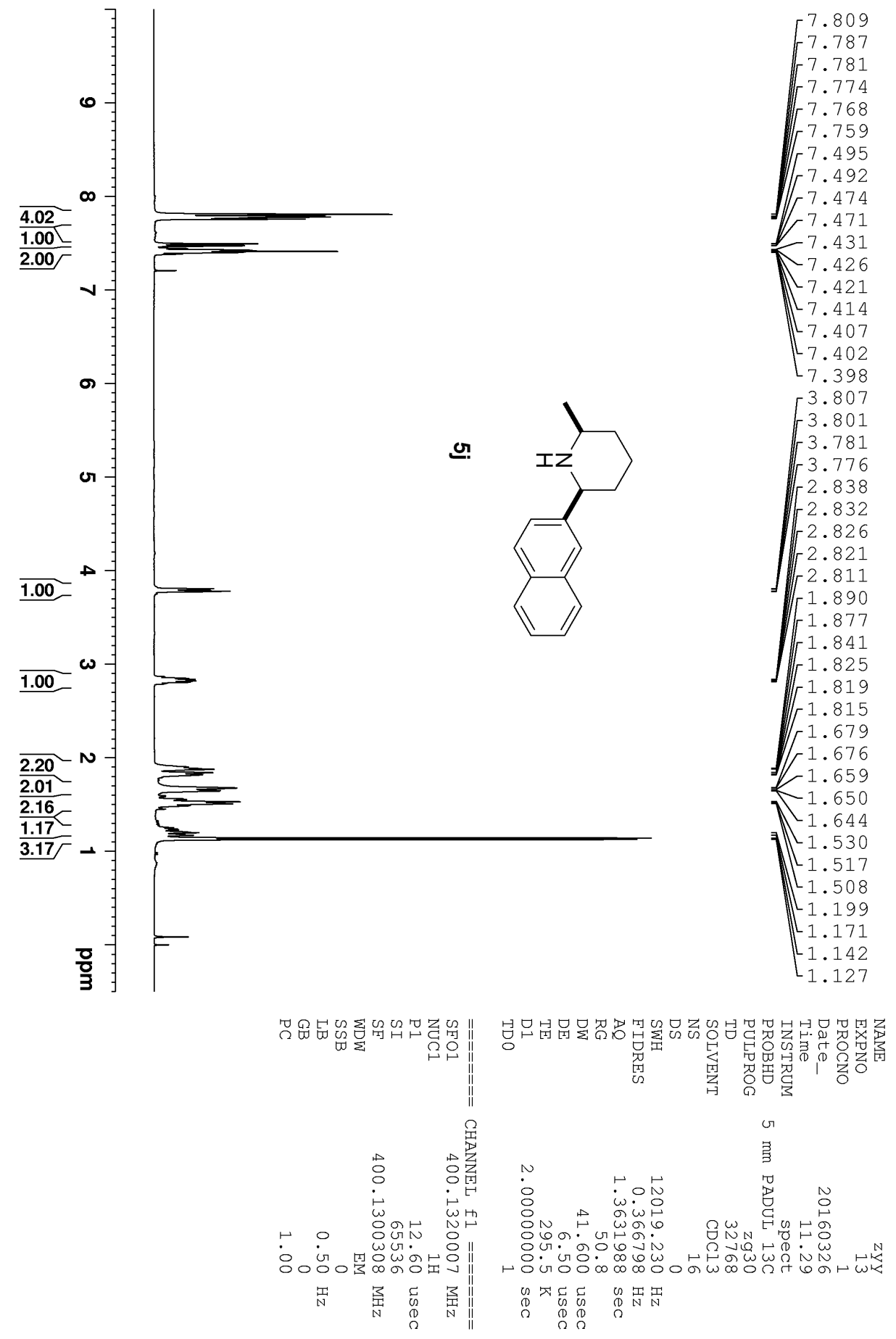



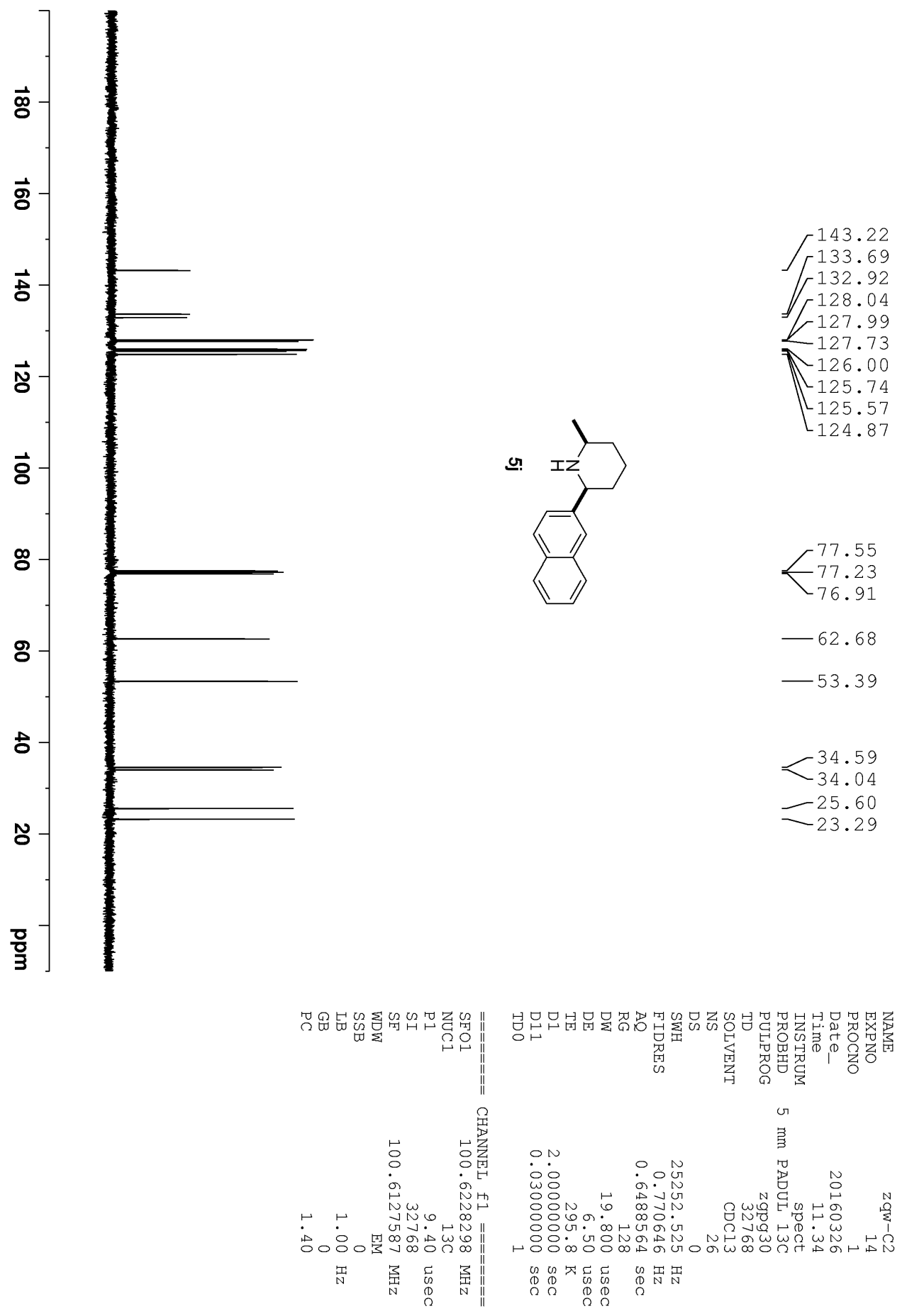


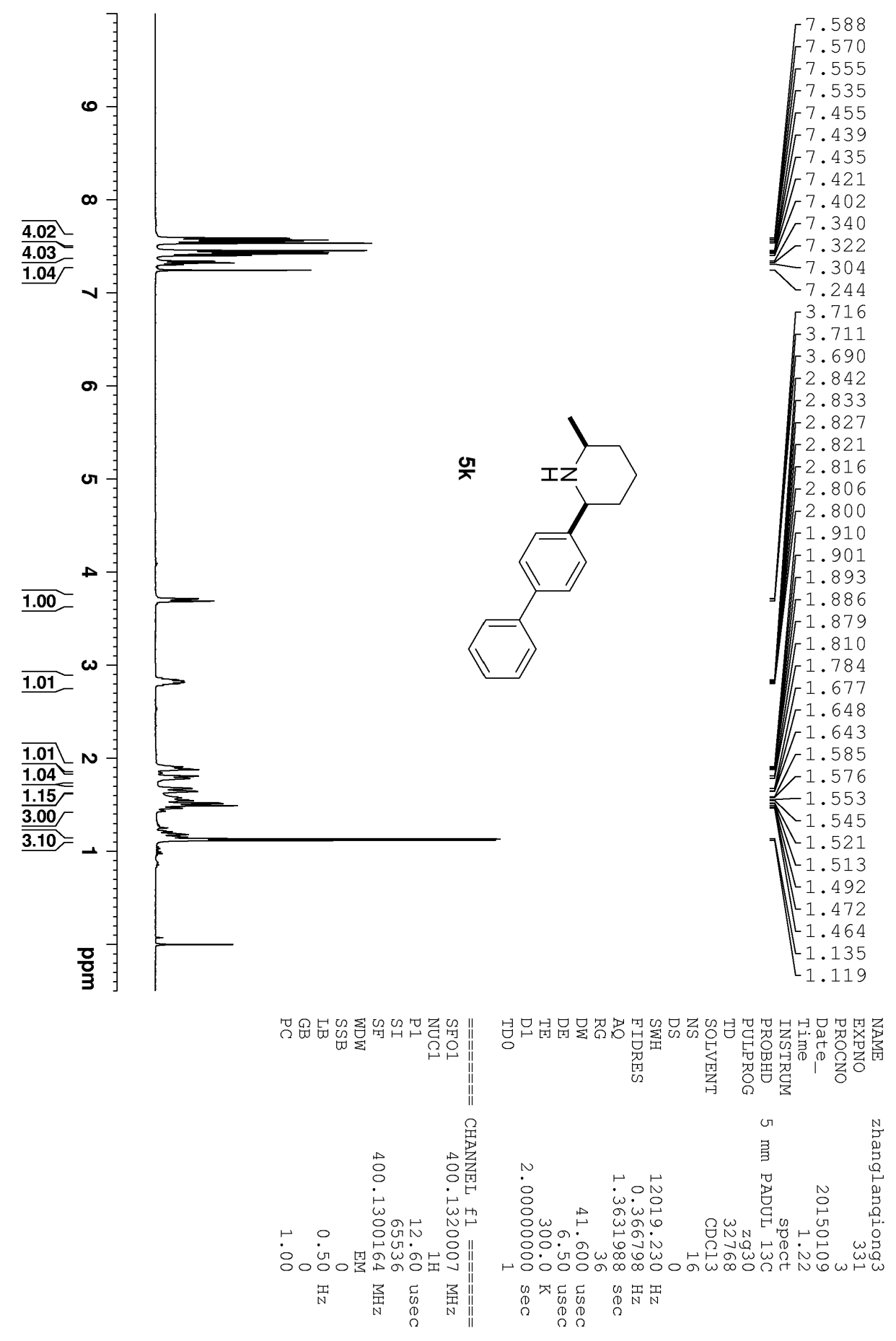




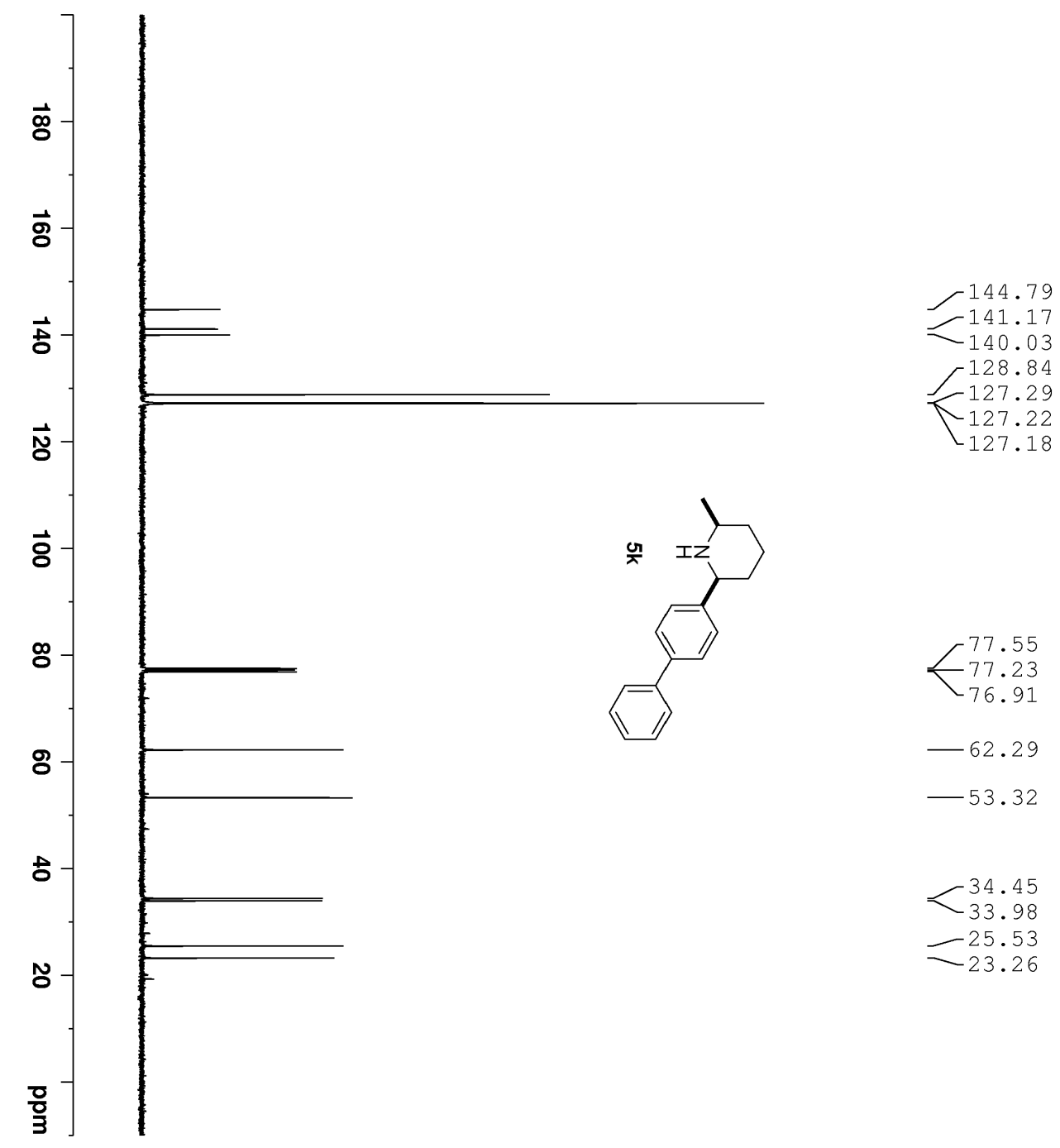

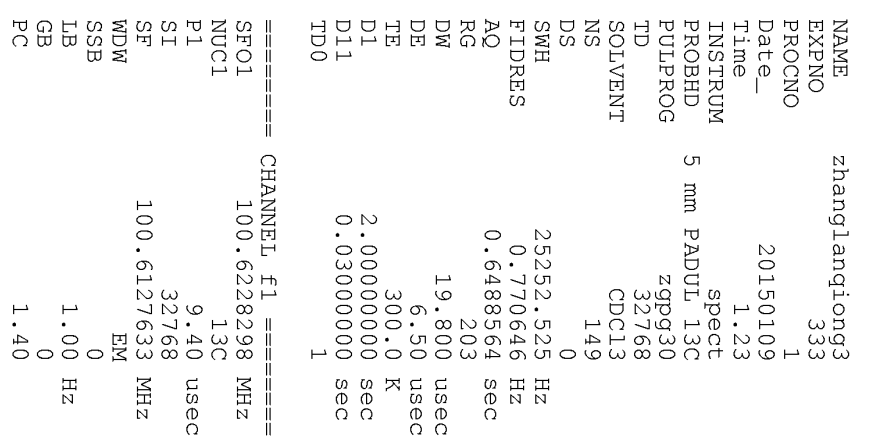




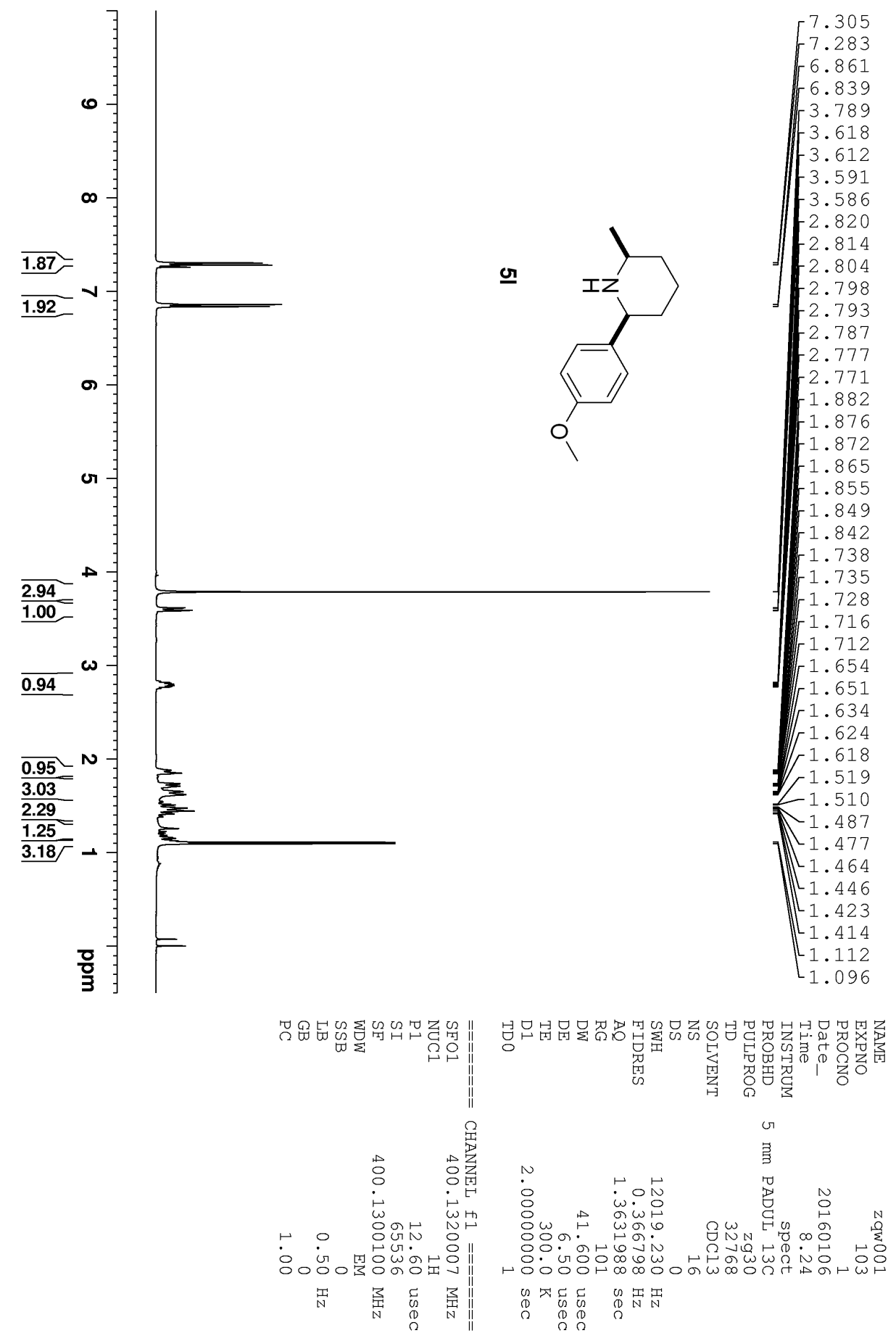




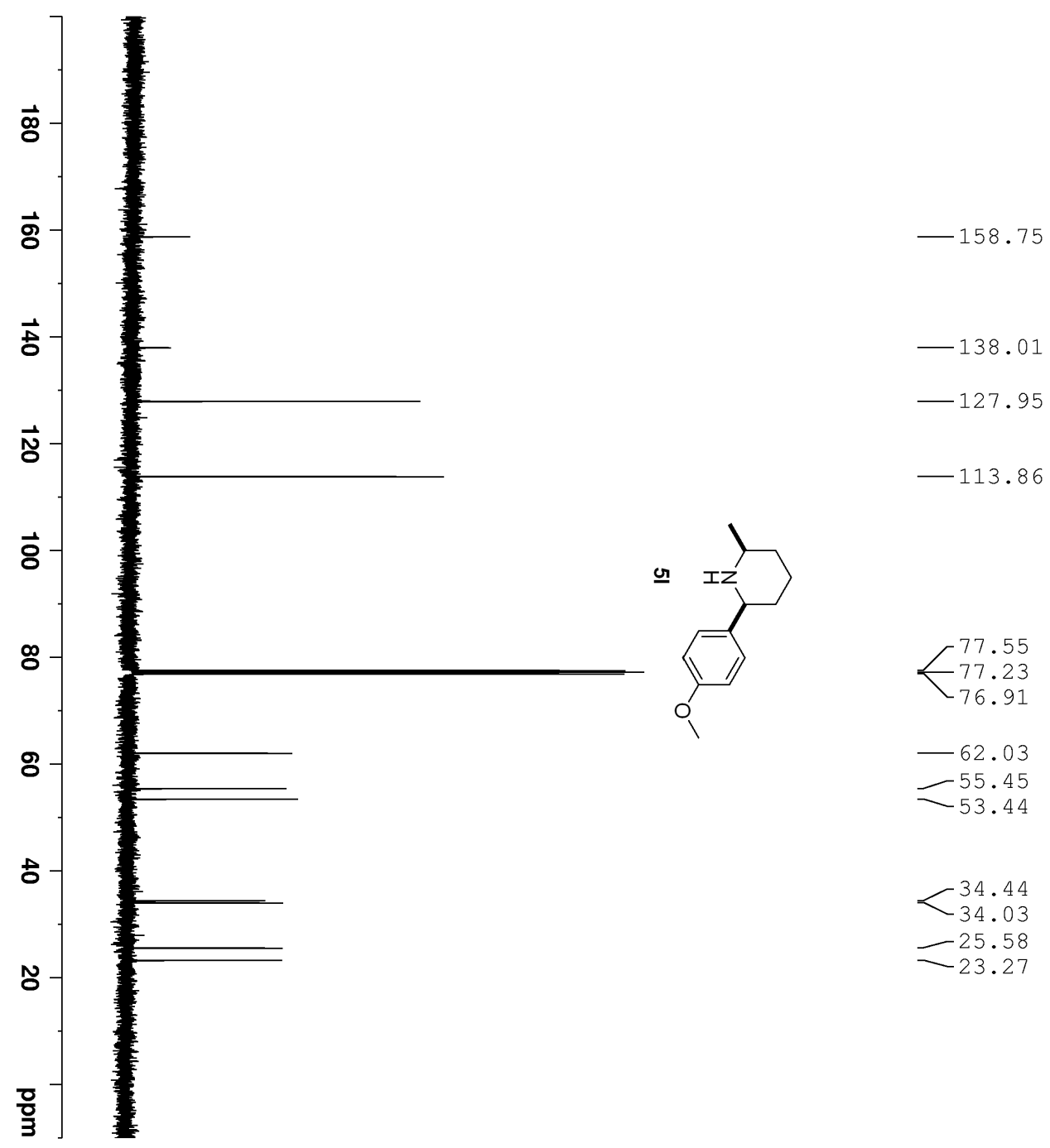

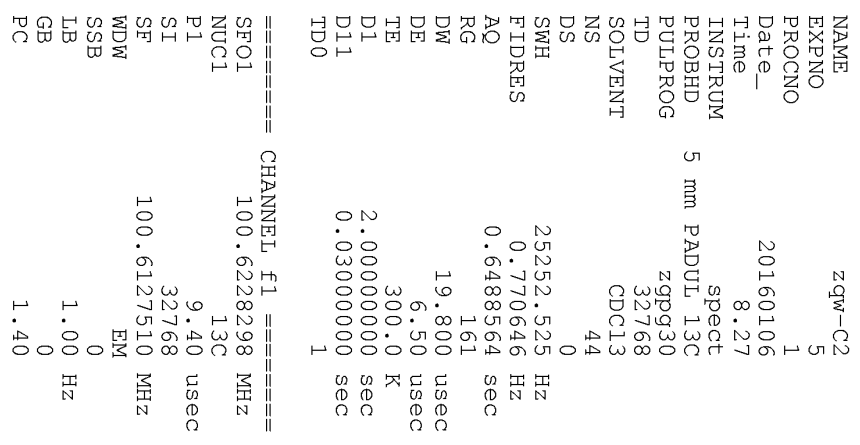




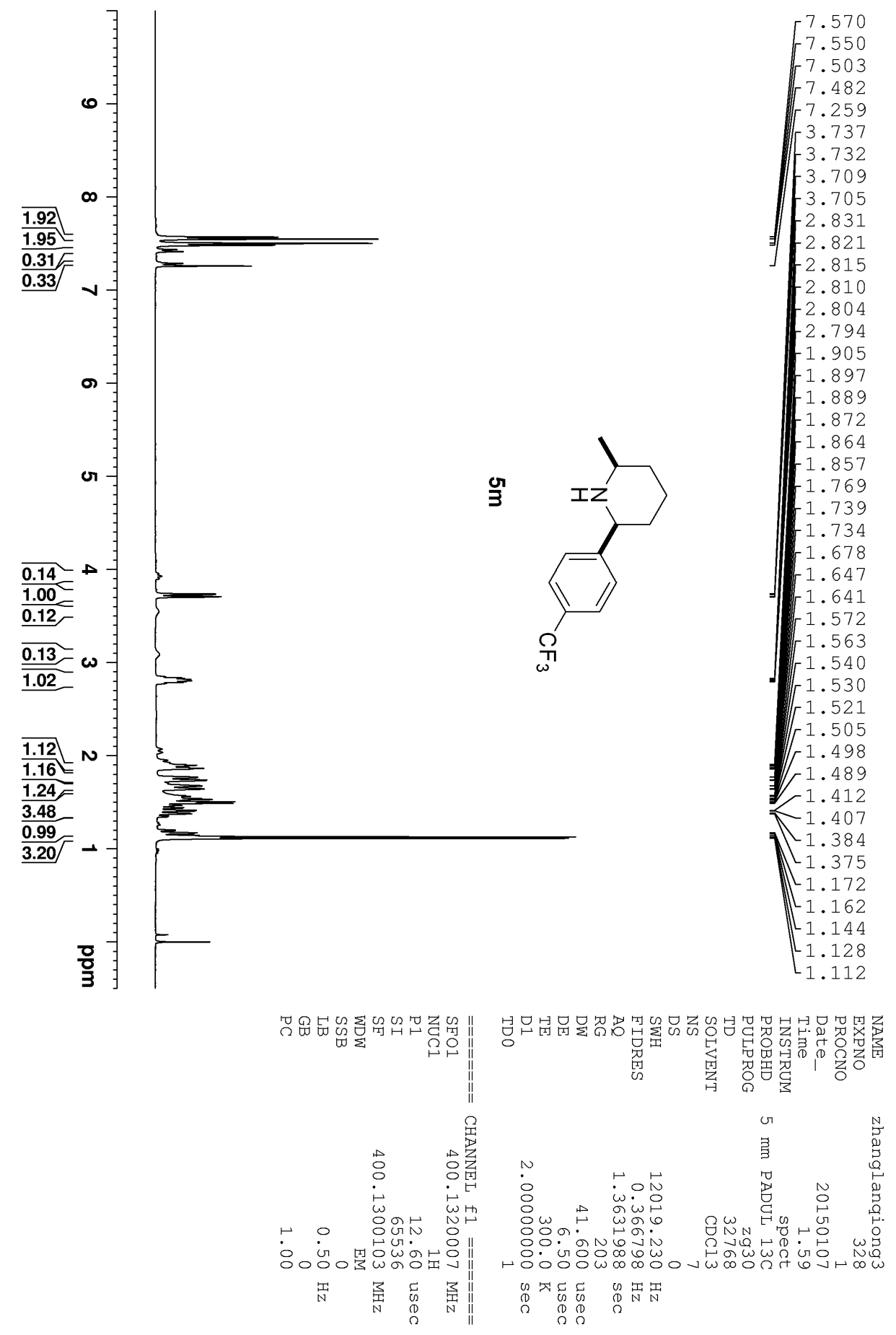




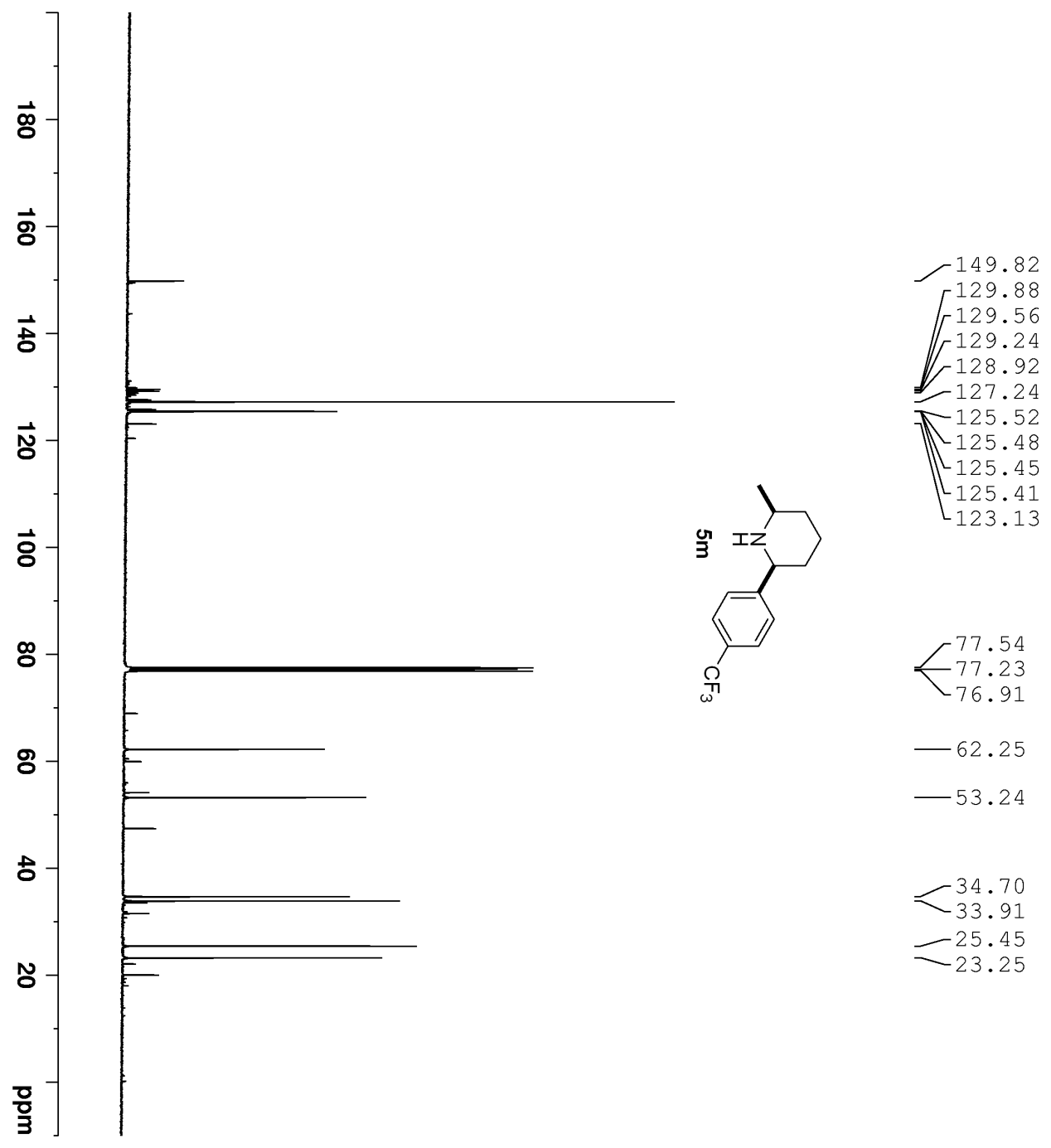

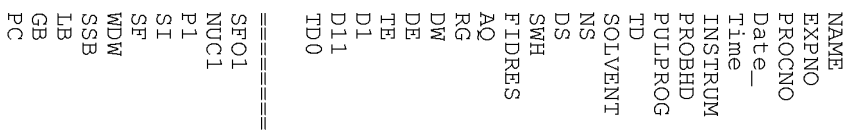

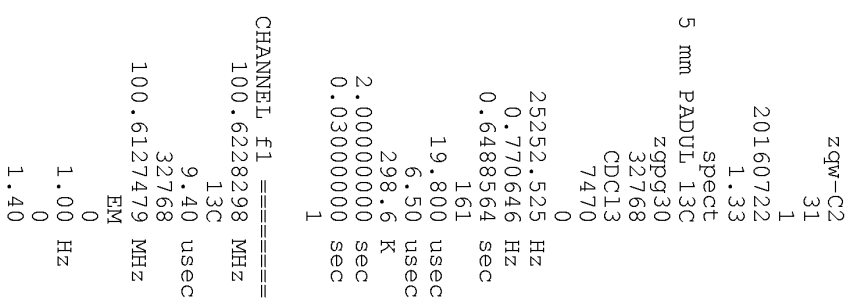




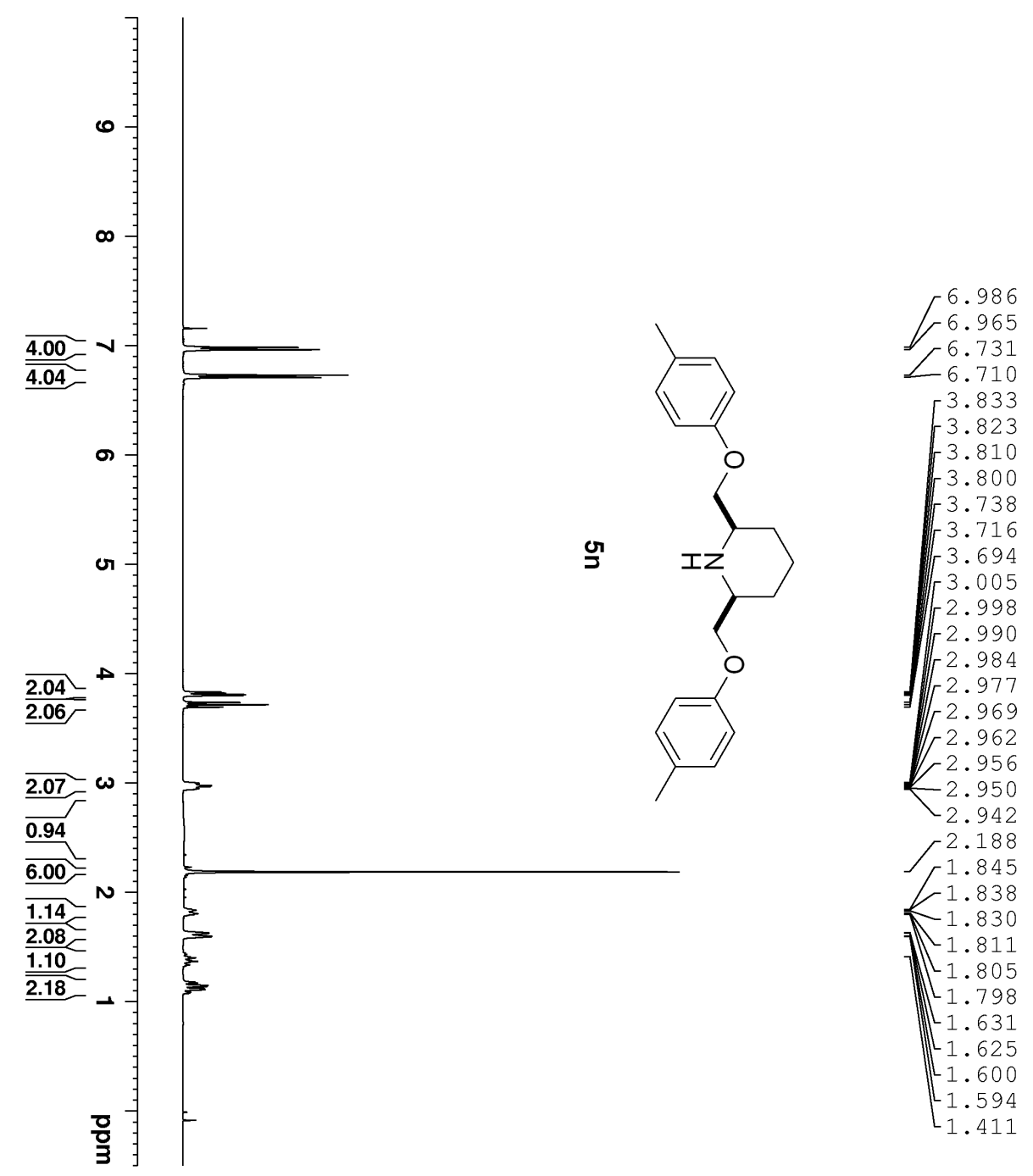

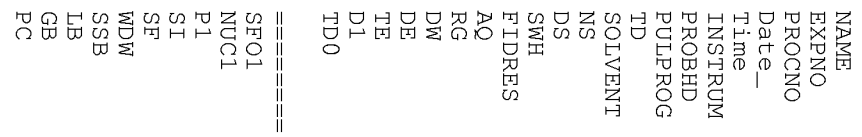

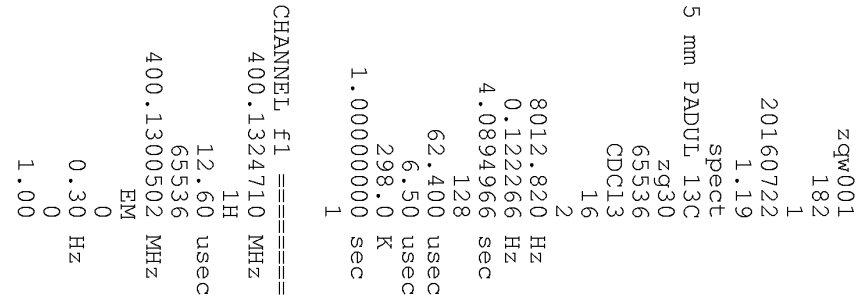



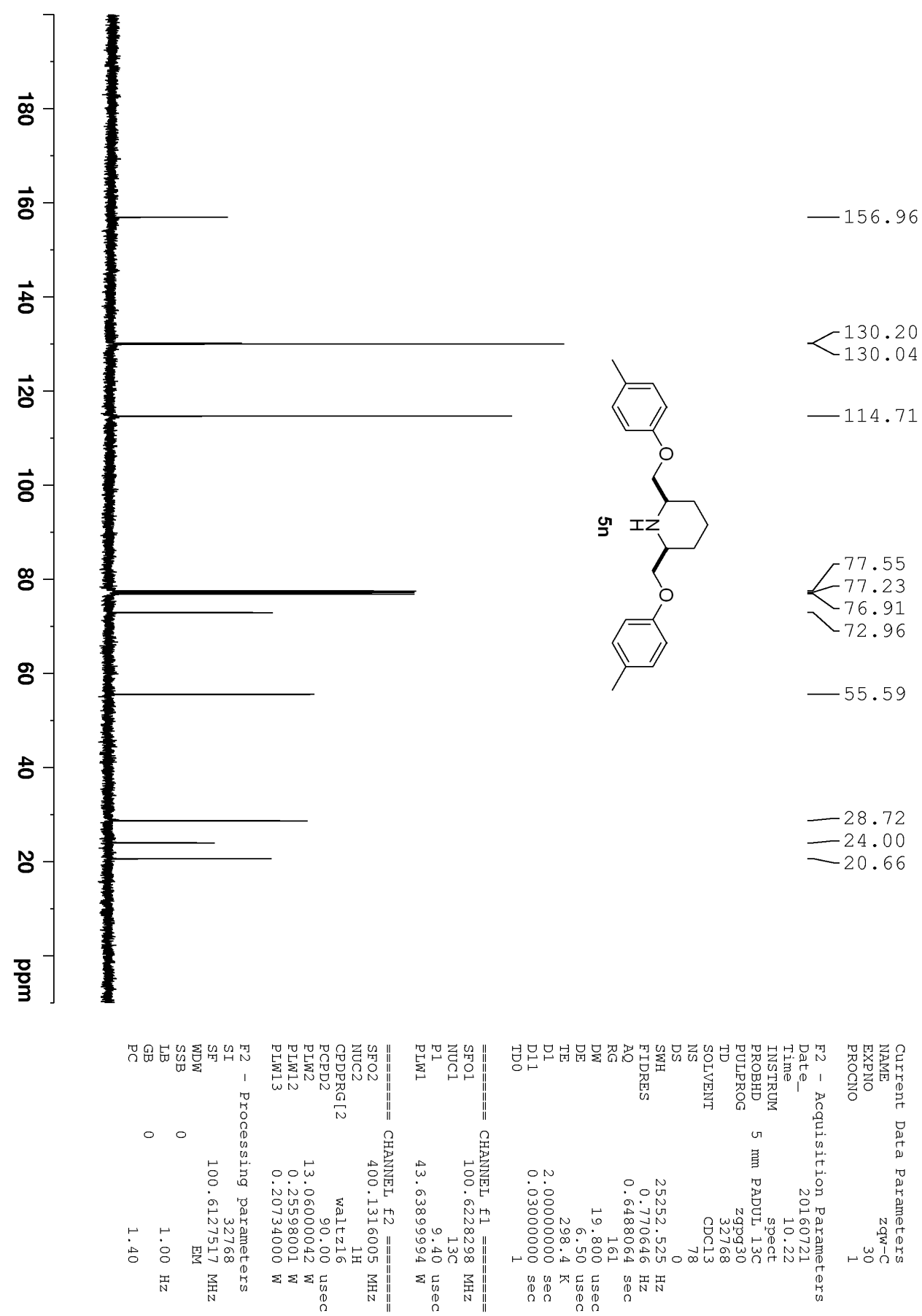


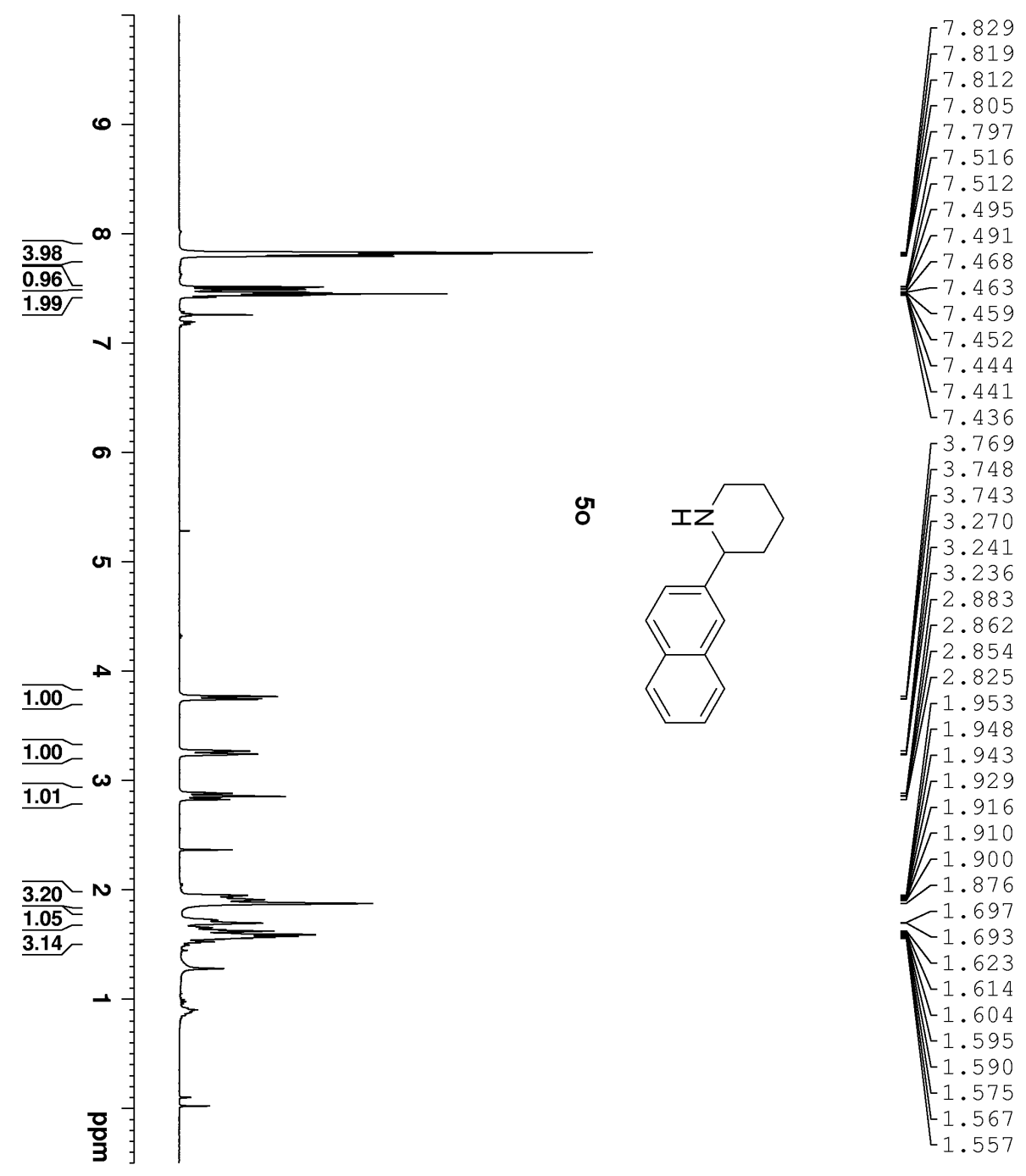

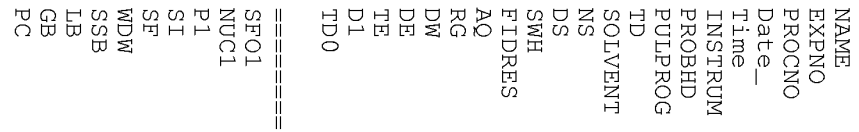

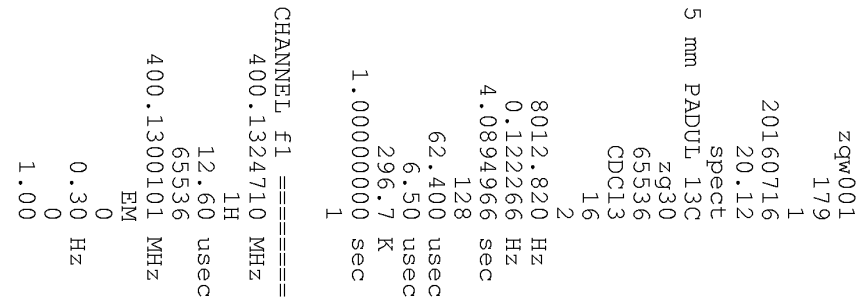




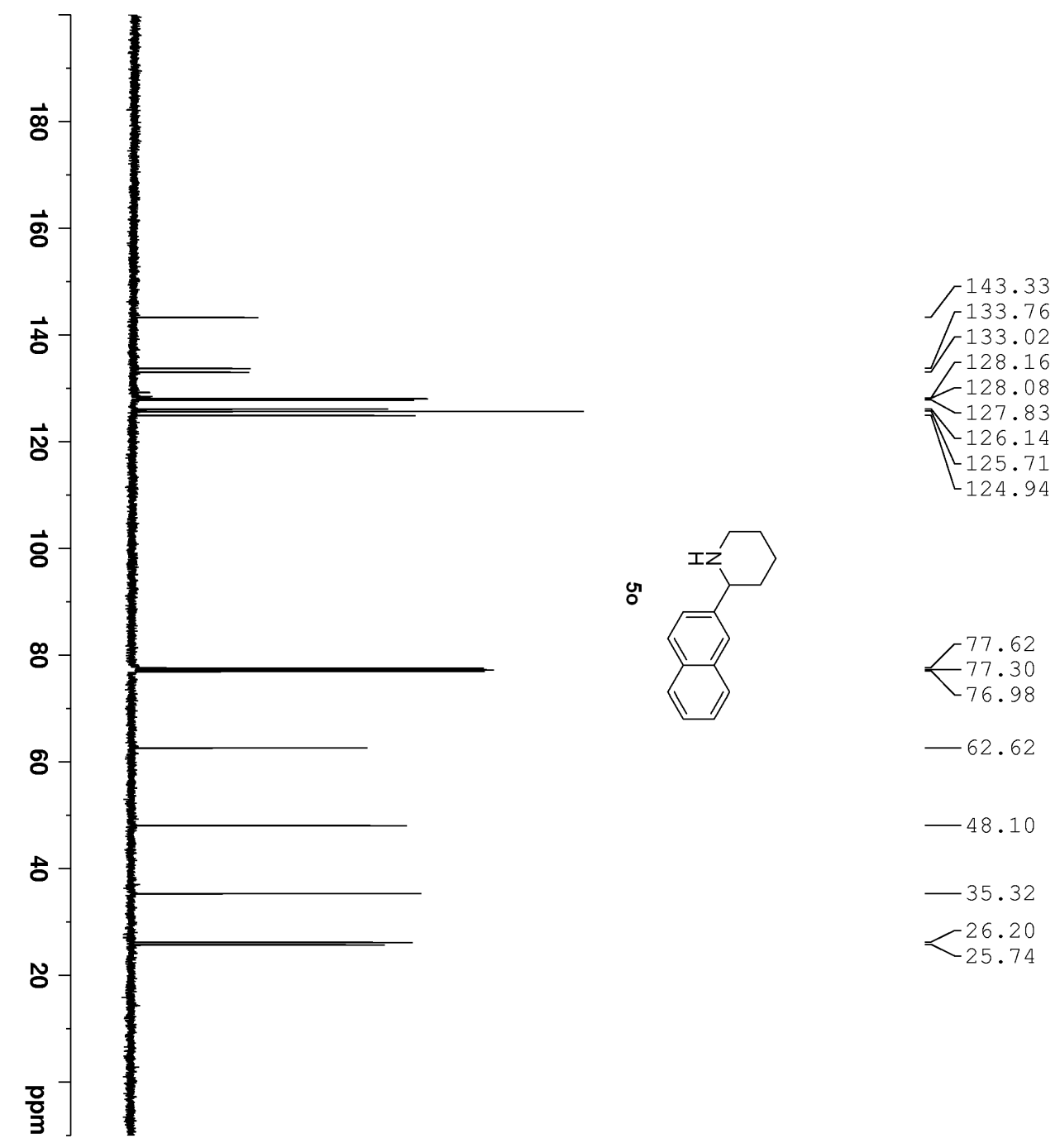

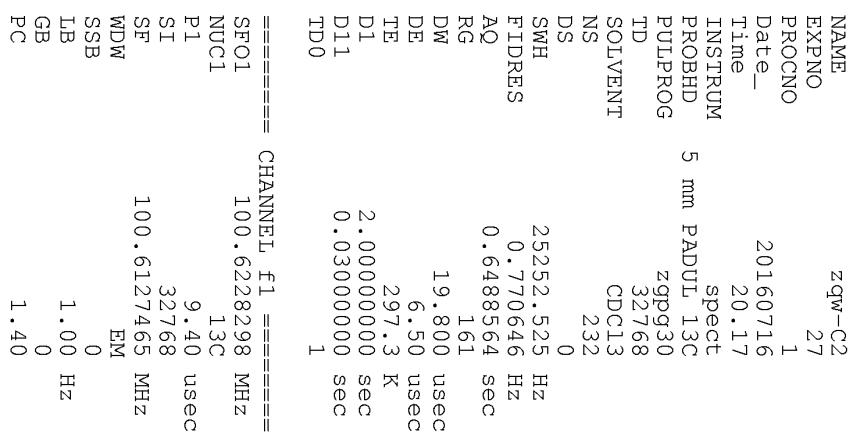




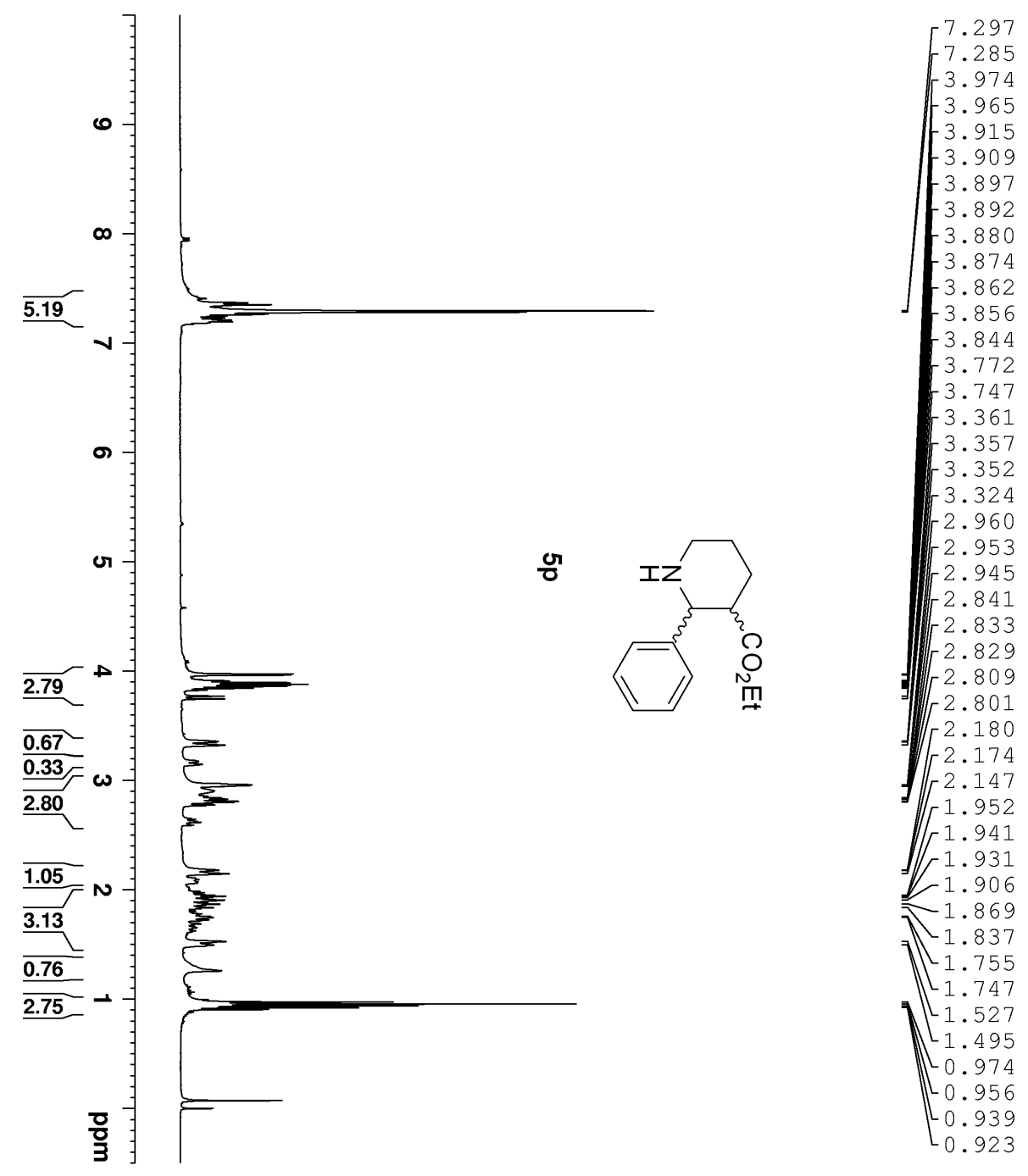

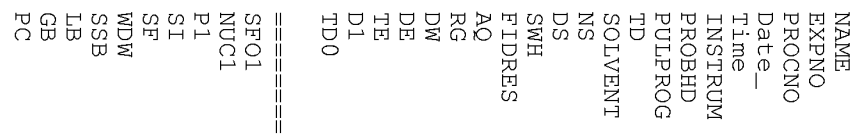

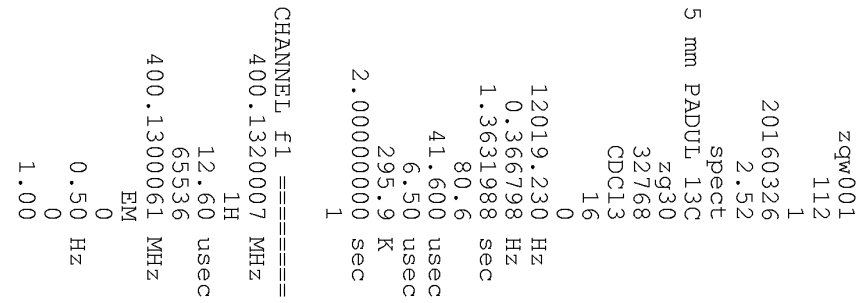




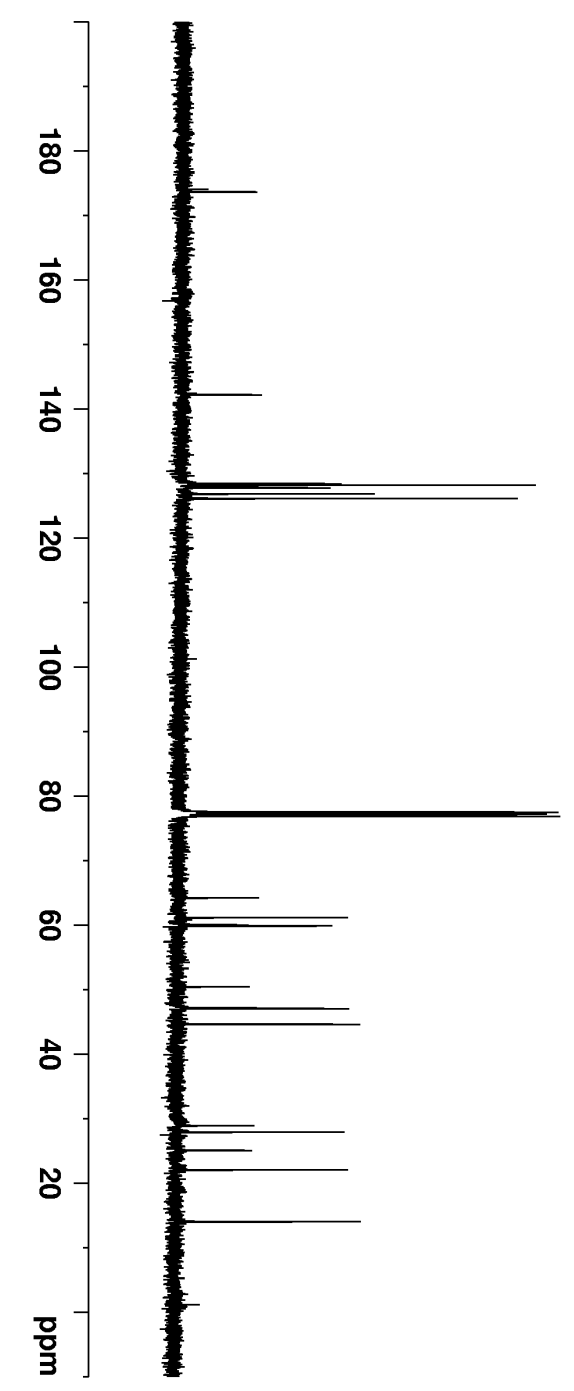

$-173.70$
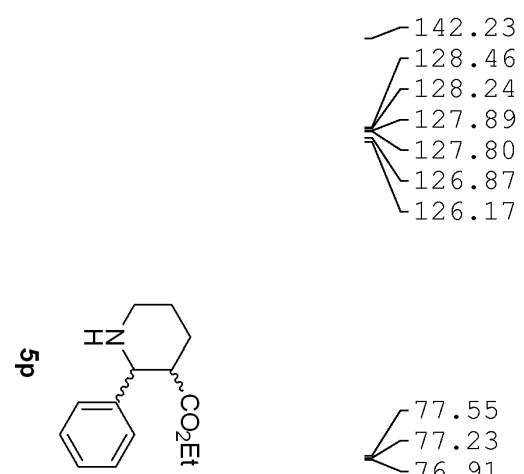

77.55
$<77.23$
-76.91

$\Gamma^{61.20}$

59.91

$-50.46$

47.21

47.11

44.64

$-28.94$

$-27.95$

$-25.12$

$-14.07$

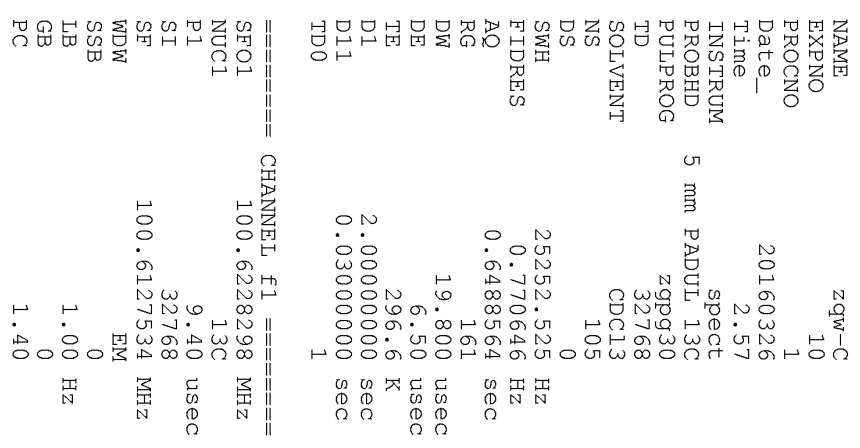




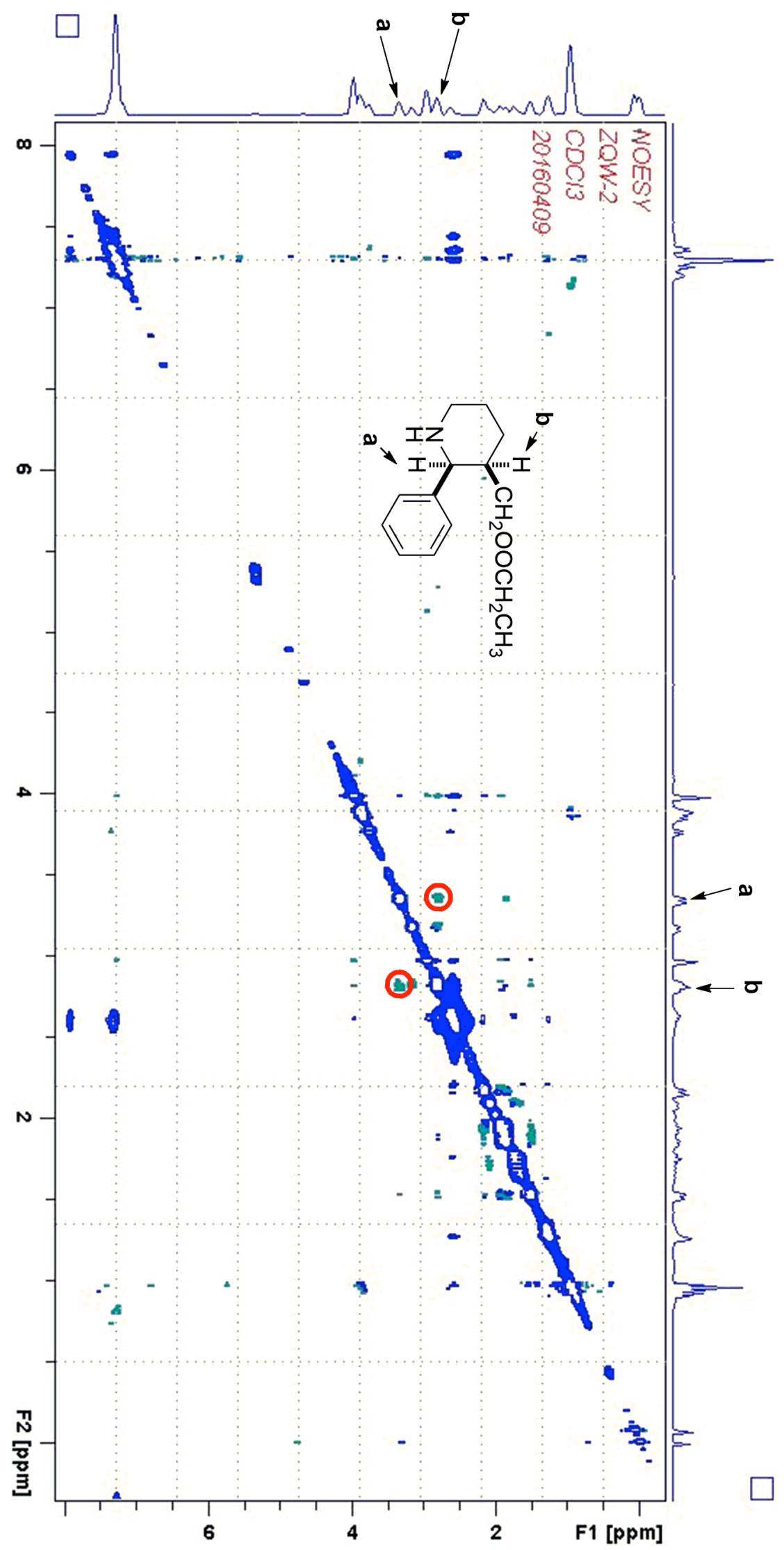




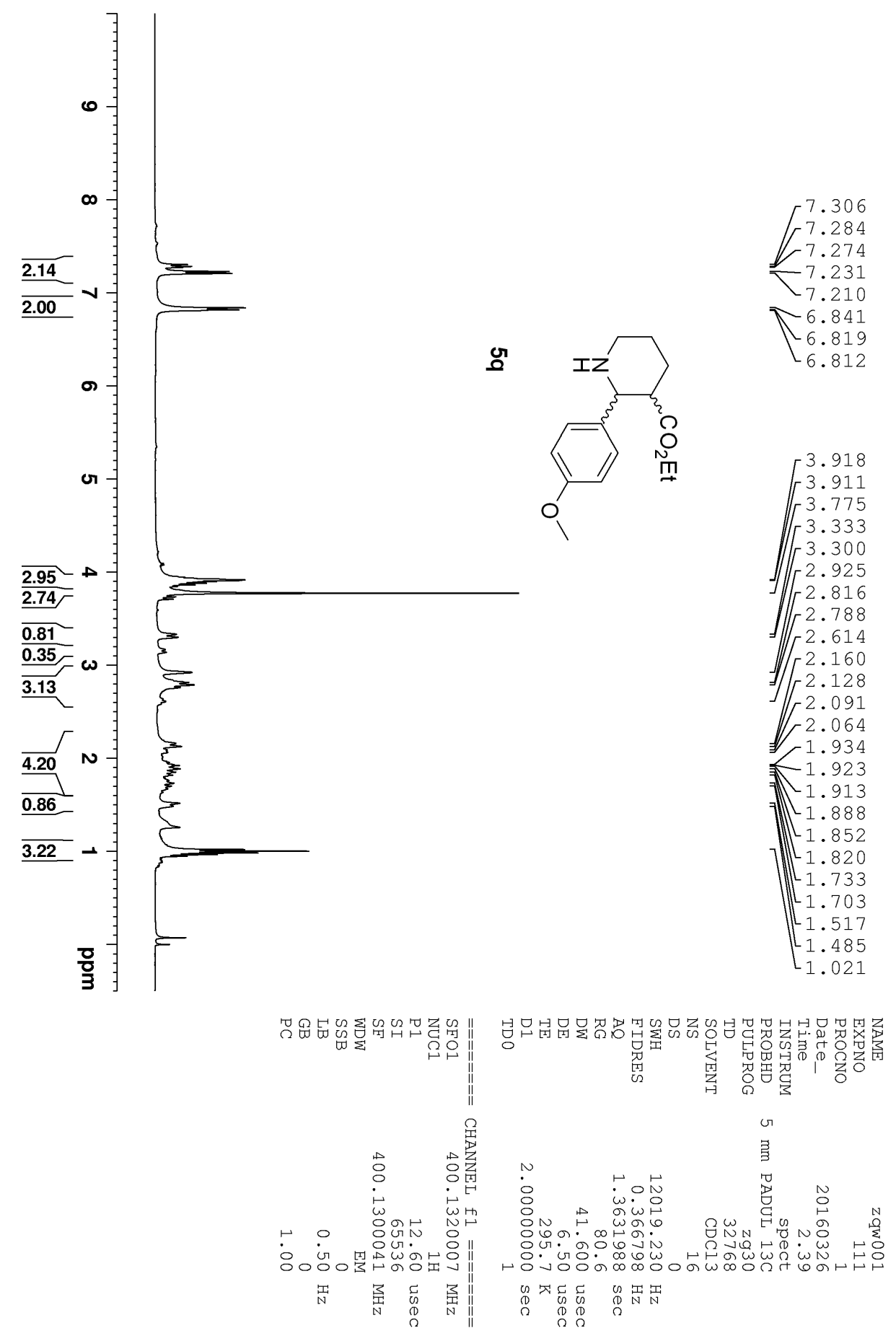




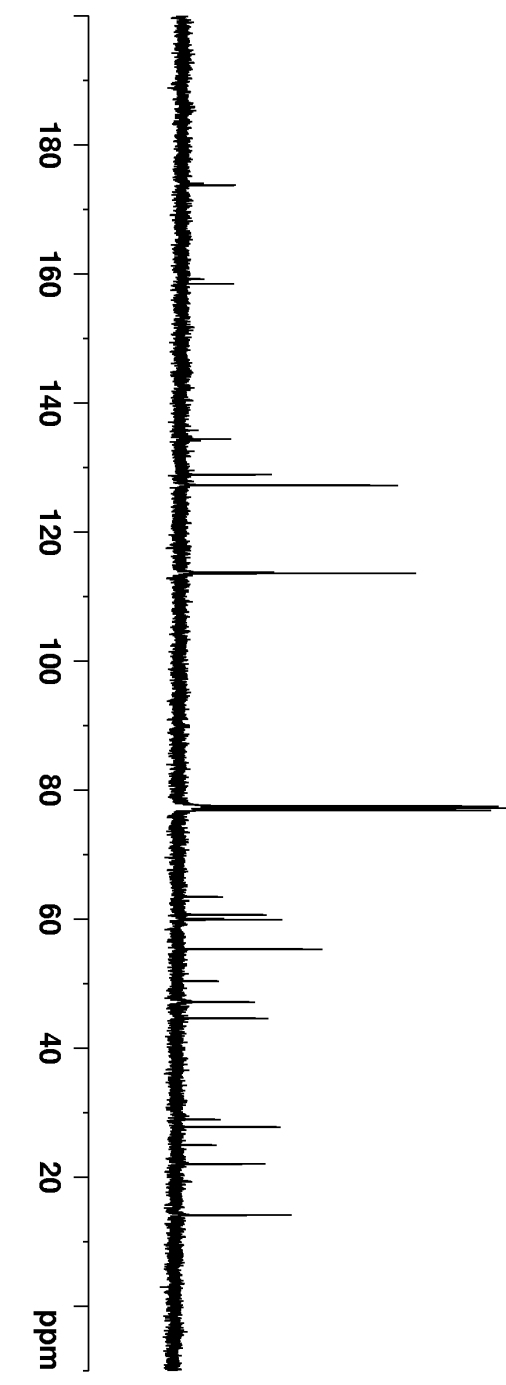

$-173.78$

$-158.50$

$-134.44$

$-128.92$

$-127.27$

113.80
$<113.61$

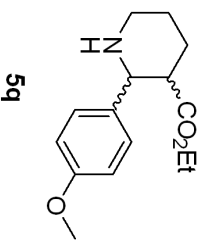

$\begin{array}{r}77.55 \\ -77.23 \\ \hline 76.91\end{array}$

$-76.91$

63.49

60.72

60.10

59.93

55.37
-

$-50.45$

$7-47.23$

47.15

44.64

$-28.96$

$-27.83$

$-25.00$

-22.08
-14.16

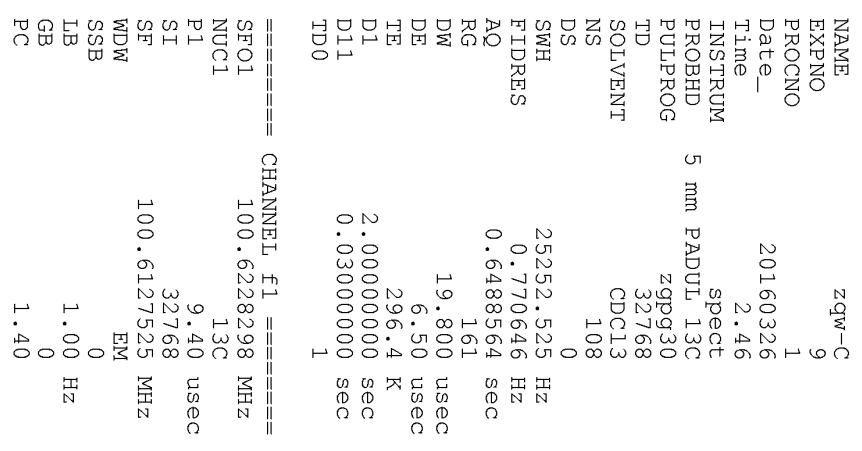




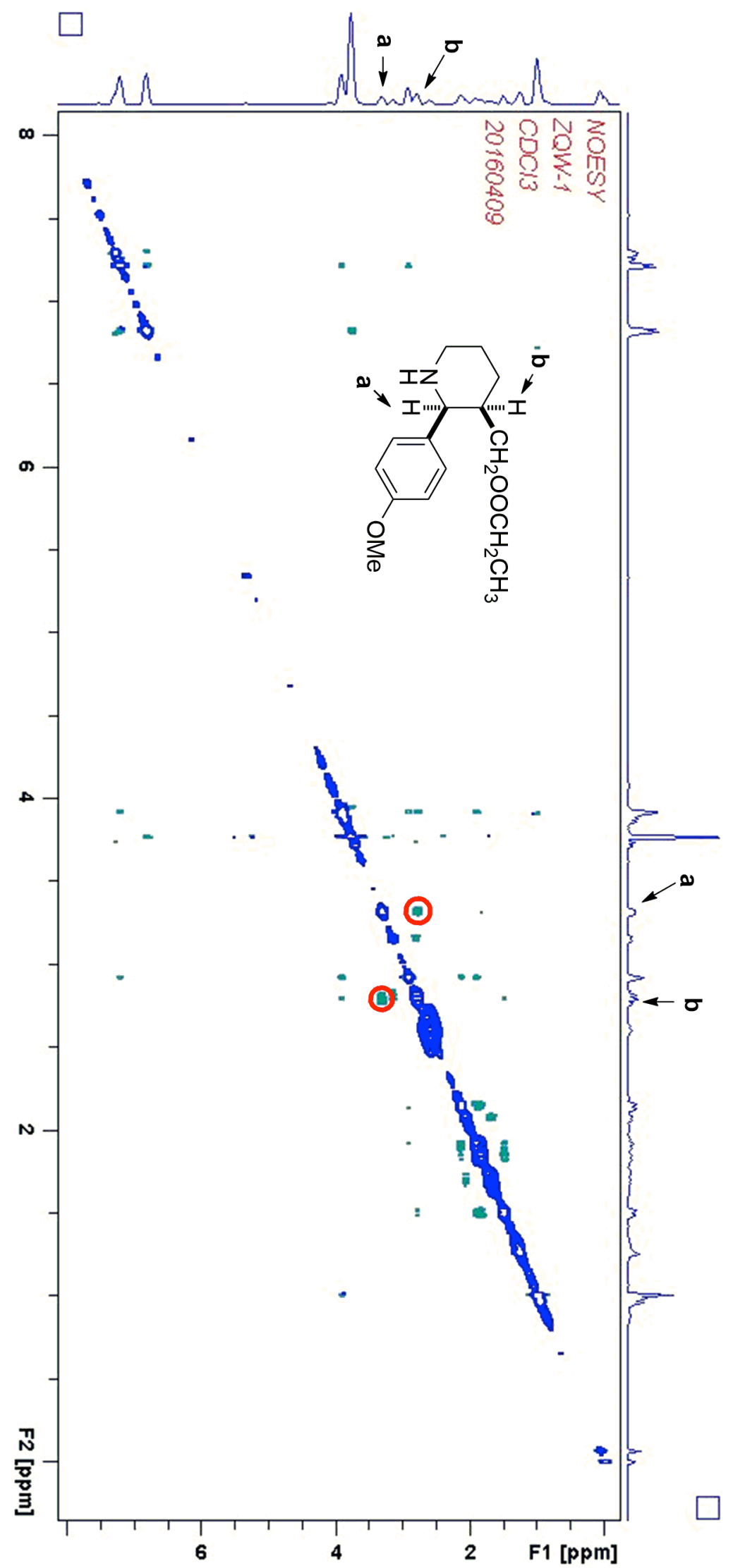

